\title{
Redes de projeções por inversão: propriedades simétricas do sistema cromático e equivalências por multiplicação $\mathrm{M}_{5}$ e $\mathbf{M}_{7}$
}

Inversional Projections Network: symmetrical properties of the chromatic system and equivalences by multiplication

$\mathrm{M}_{5}$ and $\mathrm{M}_{7}$

Joel Albuquerque (USP) joelalbuquerque@usp.br 


\section{Resumo}

Este trabalho é o segundo momento de reflexões sobre a rede de projeções por inversão, um conceito apresentado anteriormente em nosso artigo "Rede de Projeções por Inversão, Relações entre Tonnetze de Diferentes Tricordes" (ALBUQUERQUE e SALLES: 2017). Retomaremos a nosso estudo sobre este esquema de organização harmônica que foi desenvolvido a partir da necessidade de encontrar um modelo que pudesse relacionar conjuntos de classes de alturas de distintas espécies e diferentes cardinalidades, uma demanda aparentemente não contemplada por propostas neorriemannianas tradicionais. Nosso sistema foi construído calcado nas concomitâncias entre conjuntos implícitos em diferentes Tonnetze gerados a partir de inversões de todas as possibilidades de tricordes, revelando importantes propriedades simétricas implícitas em uma ampla extensão no universo cromático. Nosso trabalho se ampara na discussão promovida por teóricos dedicados ao desenvolvimento de ferramentas analíticas especializadas para o estudo do repertório pós-tonal, se inclinando em particular para o consórcio entre parâmetros oriundos da teoria dos conjuntos e da teoria neorriemanniana.

Palavras-chave: Rede de projeções por inversão. Simetria. Teoria dos conjuntos. Teoria neorriemanniana. Música pós-tonal.

\section{Abstract}

This work is a second reflection on the Inversional Projections Network, a concept presented previously in our article "Inversional Projections Network, Relationship between Tonnetze of Different Tricords" (ALBUQUERQUE, SALLES: 2017). We return here to our study of the scheme of harmonic organization developed to supply a model that could relate sets of different species and different cardinalities, a demand apparently not contemplated by traditional neo-Riemannian proposals. We built our system based on the concomitants between sets implicit in different Tonnetze, generated from inversions of all possibilities of tricords, revealing important symmetrical properties inherent to the chromatic universe in its full extension. We worked supported by the extensive discussion promoted by theorists dedicated to the development of specialized analytical tools for the study of the post-tonal repertoire, focusing in particular on the consortium between parameters derived from musical set theory and neo-Riemannian theory.

Keywords:Inversional projections network. Symmetry. Musical set theory. Neo-Riemannian theory. Post-tonal music. 


\section{Rede de projeções por inversão1 e equivalências por multiplicação M5 e M7}

A partir do anterior levantamento de confluências entre diversos Tonnetze diferentes construídos a partir de tricordes, construímos um sistema de projeções por inversões que apenas relacionava tetracordes (Mod4), estes projetados a partir de díades de alturas (Mod2) presentes como subconjuntos de tricordes (Mod3). Ampliamos este sistema ao incluir a relação entre pentacordes projetados a partir de alturas avulsas (Mod1), estas selecionadas como subconjuntos dos mesmos tricordes e representadas pelas arestas dos triângulos que aparecem nas figuras, acrescentando também os tetracordes e tricordes implícitos que projetam os mesmos pentacordes. Nesta estrutura percebemos que cada tricorde se conecta com outros seis conjuntos projetados por inversão: três em torno de suas vértices e três em torno de suas arestas. A partir desta constatação, seguimos ampliando esta rede de conjuntos relacionados por projeções a partir de inversões, como podemos verificar na figura abaixo (Fig.1).

1 Conferir a primeira parte da discussão sobre a construção deste sistema no artigo "Rede de Projeções por Inversão, Relações entre Tonnetze de Diferentes Tricordes" (ALBUQUERQUE e SALLES, 2017). Também sugerimos a leitura de alguns textos fundamentais que apresentam parâmetros importantes sobre as ferramentas de análise pós-tonal discutidas em nossa pesquisa. Sobre teoria dos conjuntos e alguns de seus conceitos - classes de conjuntos intervalares, transposição (Tn) e inversão (Tnl) de grupos de alturas, e ciclos intervalares - ver FORTE, 1988; STRAUS, 2005. Sobre teoria neorriemanniana e teoria transformacional, esta segunda uma vertente decorrente da anterior, verificar LEWIN, 1982 e 1987; COHN 1998a, 1998b e 2012; DOUTHETT e STEINBACH, 1998; GOLLIN, 1998, TYMOCZKO, 2007, 2009 e 2011. Sobre a teoria dos ciclos conferir PERLE, 1977 e 1996; FOLEY, 1998. Sobre a inter-relação entre estas três correntes teóricas em torno de resultados comuns, consultar MORRIS 1982 e 2007. Sobre a representação algébrica da simetria intervalar por matrizes de soma e multiplicação, e a verificação de correlações por invariâncias entre grupos de alturas, ver BABBITT, 1960 e 1961; LEWIN, 1982. Sobre a relação entre conjuntos intervalares por multiplicação; multiplicação de um grupo de alturas por um índice numérico invariante (Mn); e "combinação transpositiva", conferir COHN, 1988; BOULEZ, 2011; OLIVEIRA, 2007; RAHN, 1980; SCOTTO, 2014; YUST, 2015. Sobre aspectos da simetria a partir de uma perspectiva matemática, ver WEYL, 1997 e STEWART, 2012. Sobre conjuntos intervalares simétricos, eixos de simetrias, somas de eixo, inversões de conjuntos e a representação geométrica da simetria por clockface, ver ANTOKOLETZ, 2006; STRAUS, 2005. 


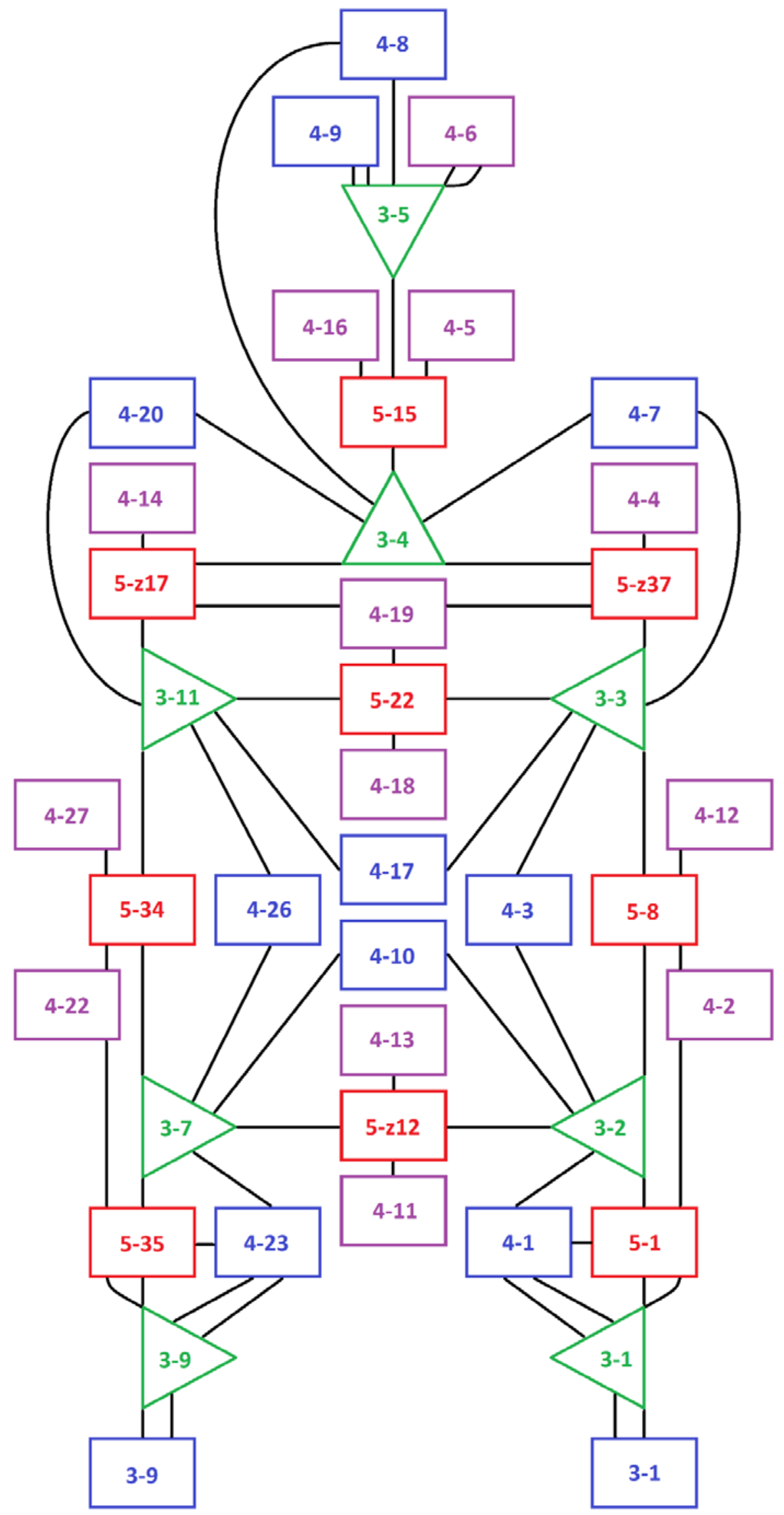

Fig. 1: Ampliação da rede de projeções por inversão de tricordes 
Podemos perceber que esta grande estrutura de quarenta e dois conjuntos relacionados por projeções a partir de inversões apresenta propriedades simétricas em diversos níveis, com destaque para a equivalência entre os dois grandes grupos de treze conjuntos cada que estão nas laterais esquerda e direita da figura. Ambos os grupos se correspondem verticalmente em número de conjuntos, conjuntos com o mesmo índice de alturas em posições similares e conexões idênticas entre os elementos que configuram cada estrutura. Outra característica importante é que nas extremidades onde se limitam os desdobramentos de cada grupo (na parte inferior da figura) temos três conjuntos equivalentes que são recordes de ciclos intervalares: C5 (ordem da classe de intervalo ${ }^{2}|5|$ ) à esquerda (conjuntos 3-9, 4-23 e 5-35) e C1 (ordem da classe de intervalo |1|) à direita (conjuntos 3-1, 4-1 e 5-1). Esta disposição nos faz perceber que cada estrutura é construída a partir de inversões e projeções a partir dos ciclos intervalares C5 e C1, assim escolhemos identificar os respectivos grupos de conjuntos como região C5 (a esquerda) e região C1 (a direita) (Fig.2). 

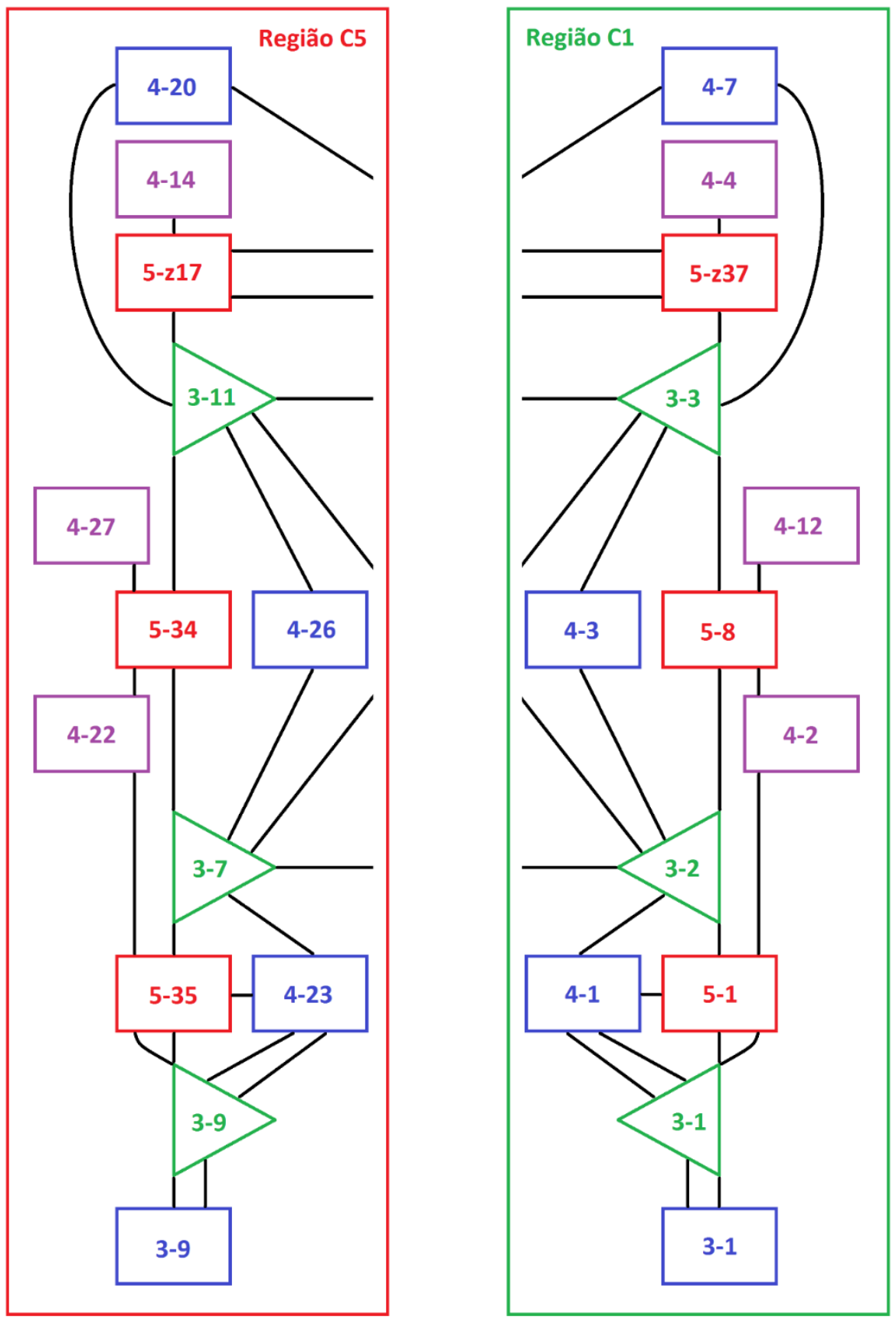

Fig. 2: Regiões C1 e C5 da rede de projeções por inversão

Procuramos mais detalhes sobre esta relação entre essas duas regiões C1 e C5 e identificamos que existe uma comum invariância entre os índices de vetores intervalares dos conjuntos correspondentes. Verificamos que os vetores que aparecem reiterados são as entradas dois, três, quatro e seis, sendo que os vetores um e cinco aparecem invertidos entre si em todos os casos (Tab.1). Esta constatação nos revela que os conjuntos da região C5 podem ser obtidos por multiplicação pelos índices M5 ou M7 dos seus equivalentes da região $\mathrm{C} 1$, e vice-versa, seguindo aqui o teorema proposto por John Rahn (1980: 104). 
TEOREMA: M5 e M7 têm exatamente o mesmo efeito no conteúdo intervalar. As multiplicidades de intervalos não ordenados 1 e 5 são trocadas entre si e as outras multiplicidades são mantidas (RAHN, 1980: 104)

Esta condição foi explicada em maiores detalhes por João Oliveira (2007: 116-17).

Resumindo, o vetor intervalar de um conjunto-imagem resultante da aplicação de M5 tem as multiplicidades dos intervalos-classe 1 e 5 trocadas em relação ao conjunto original; todas as outras multiplicidades permanecem inalteradas (OLIVEIRA, 2007: 116).

Tal como acontece em M5, sendo os intervalos $<1>\mathrm{e}<11>$ pertencentes ao intervalo-classe ic $=|1|$, e os intervalos $<5>$ e $<7>$ pertencentes a ic $=|5|$, as multiplicidades dos intervalos-classe 1 e 5 trocam entre si após M7. Todas as outras multiplicidades permanecem inalteradas (OLIVEIRA, 2007: 117).

No caso de um conjunto Z, os operadores M5 e M7 por vezes transformam esse conjunto no seu correspondente $Z$ que, conforme foi explicado, possui um vetor intervalar idêntico (OLIVEIRA, 2007: 117).

Exemplos:

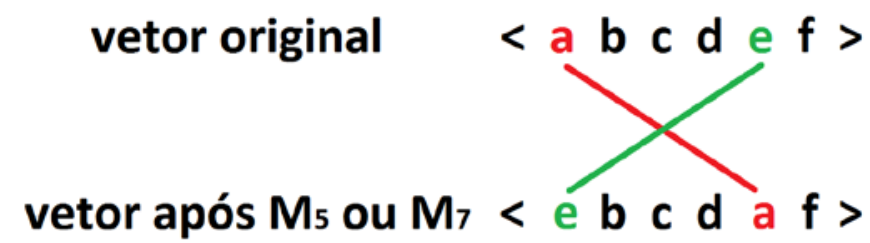

$$
\begin{aligned}
& K=[0,2,5,8] \\
& M 5(K)=(0,10,1,4)=[0,2,3,6]
\end{aligned}
$$

\section{4-27vetor $\langle 012110\rangle$}

4-12 vetor $<112101>$

$K=[0,2,4,6,9]$

$M_{7}(K)=(0,2,4,6,3)=[0,2,3,4,6]$

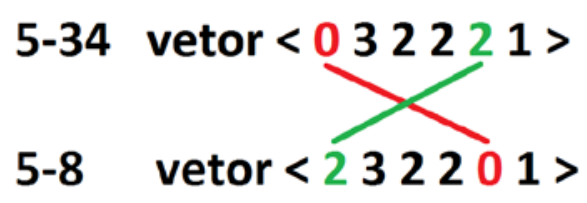


Relação arestas

\begin{tabular}{|c|c|c|c|}
\hline Região C5 & Vetores & Região C1 & Vetores \\
\hline $4-14$ & {$[111120]$} & $4-4$ & {$[211110]$} \\
\hline $5-z 17$ & {$[212320]$} & $5-z 37$ & {$[212320]$} \\
\hline $4-27$ & {$[012111]$} & $4-12$ & {$[112101]$} \\
\hline $5-34$ & {$[032221]$} & $5-8$ & {$[232201]$} \\
\hline $4-22$ & {$[021120]$} & $4-2$ & {$[221100]$} \\
\hline $5-35$ & {$[032140]$} & $5-1$ & {$[432100]$} \\
\hline $4-23$ & {$[021030]$} & $4-1$ & {$[321000]$} \\
\hline
\end{tabular}

Relação vértices

\begin{tabular}{|c|c|c|c|}
\hline Região C5 & Vetores & Região C1 & Vetores \\
\hline $4-20$ & {$[101220]$} & $4-7$ & {$[201210]$} \\
\hline $4-26$ & {$[012120]$} & $4-3$ & {$[212100]$} \\
\hline $4-23$ & {$[021030]$} & $4-1$ & {$[321000]$} \\
\hline $3-9$ & {$[010020]$} & $3-1$ & {$[210000]$} \\
\hline
\end{tabular}

Relação tricordes

\begin{tabular}{|c|c|c|c|}
\hline Região C5 & Vetores & Região C1 & Vetores \\
\hline $3-11$ & {$[001110]$} & $3-3$ & {$[101100]$} \\
\hline $3-7$ & {$[011010]$} & $3-2$ & {$[111000]$} \\
\hline $3-9$ & {$[010020]$} & $3-1$ & {$[210000]$} \\
\hline
\end{tabular}

Tab. 1: Relação por multiplicação M5 e M7 entre as regiões C1 e C5, identificada na análise dos índices de vetores intervalares dos conjuntos correspondentes

Inclusive as classes de intervalo |1| e |5|, que são a base de construção dos dois grupos de conjuntos, também se relacionam por multiplicação, como podemos ver na relação entre os vetores intervalares destas duas estruturas (Tab.2). 


\begin{tabular}{|c|c|c|c|}
\hline $\begin{array}{c}\text { Classe de intervalo } \\
|5|\end{array}$ & Vetores & $\begin{array}{c}\text { Classe de intervalo } \\
|1|\end{array}$ & Vetores \\
\hline$(05)$ & {$[000010]$} & $(01)$ & {$[100000]$} \\
\hline
\end{tabular}

Tab. 2: Relação de multiplicação M5 e M7 entre as classes de intervalos |1 | e |5|

Poderíamos ainda incluir nesta relação os tetracordes 4-16 e 4-5, que estão listados na região superior, mas também se relacionam por multiplicação $M 5$ e $M 7$, como podemos perceber na análise dos vetores intervalares destes conjuntos (Tab.3).

\begin{tabular}{|c|c|c|c|}
\hline Região C5 & Vetores & Região C1 & Vetores \\
\hline $4-16$ & {$[110121]$} & $4-5$ & {$[210111]$} \\
\hline
\end{tabular}

Tab. 3: Relação de multiplicação M5 e M7 entre os tetracordes 4-16 e 4-5

Outra informação aparente é que a maioria dos conjuntos da região C5 têm um índice 0 na estrada um, o que indica que a ausência da classe de intervalo |1| (segundas menores e sétimas maiores) neste grupo. O mesmo acontece com a maioria dos conjuntos da região $\mathrm{C} 1$, onde temos um índice 0 na estrada cinco, o que indica a ausência da classe de intervalo |5| (quartas e quintas justas) neste grupo. Temos ainda um índice 0 na entrada seis da maioria dos conjuntos de ambos os grupos, o que revela a ausência da classe de intervalo |6| (trítono).

Voltando à rede de conjuntos projetados por inversão, temos outros dois grupos de conjuntos, que chamamos aqui de região central e região superior (Fig.3). A região central é um conjunto de oito conjuntos que está intermediando a relação entre as regiões C1 e C5, funcionando neste contexto como um eixo de simetria que existe entre os dois grupos opostos. A região superior é composta de outros oito conjuntos que estão dispostos na parte de cima do sistema. 


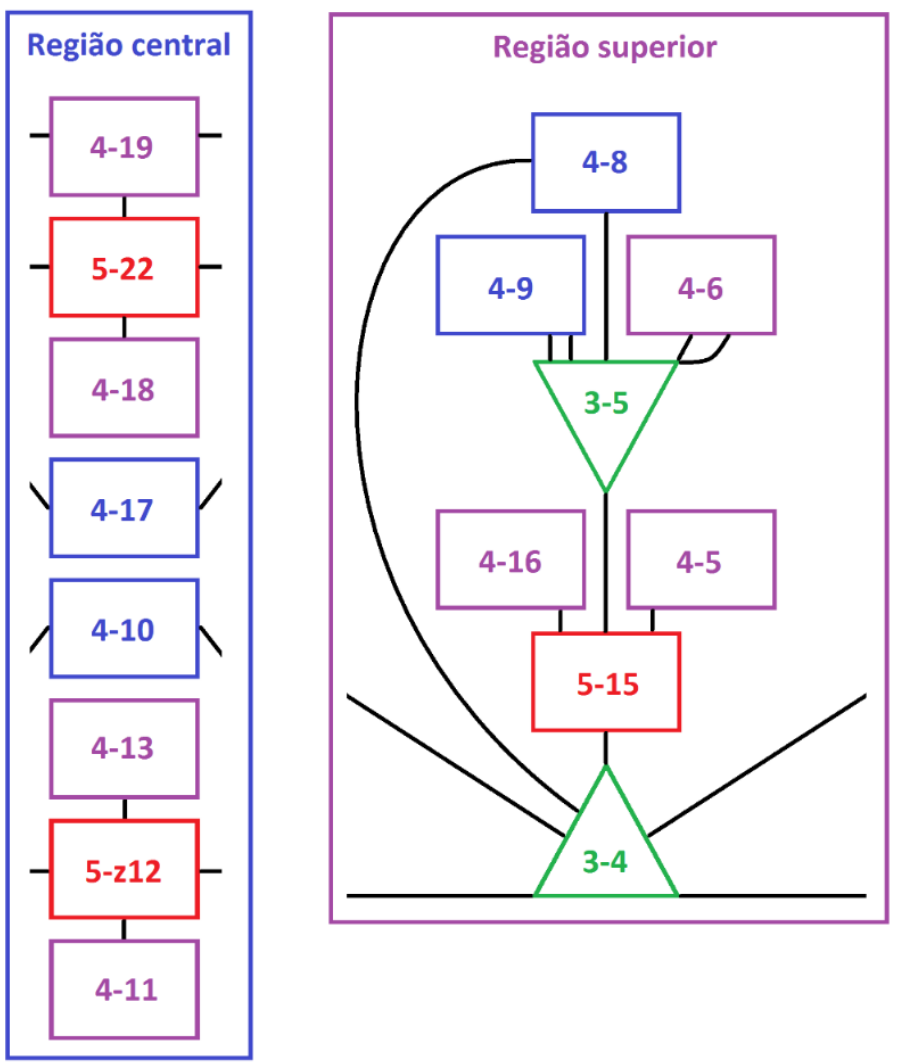

Fig. 3: Conjuntos da região central e superior

Não encontramos relações de multiplicação entre os conjuntos destes grupos, porem identificamos índices de vetores intervalares invariantes entre diversas classes de conjuntos (Tab.4). Destaque para os seis tetracordes da região central, que mantêm em comum sempre o índice 1 para as entradas um e cinco de seus respectivos vetores intervalares. No caso da região superior, temos em comum o índice 0 na entrada três dos vetores intervalares de todos os conjuntos deste grupo, o que revela a ausência da classe de intervalo |3| (terças menores e sextas maiores). Além disso, não temos nenhum caso de índice 0 na entrada seis, o que indica a presença de trítono em todos estes conjuntos, ao contrário do que aparece na maioria dos conjuntos das regiões $\mathrm{C} 1$ e C5. Outra constatação é a invariância entre as entradas um e cinco dos pentacordes 5-z37, 5-z17, 5-15, 5-22 e 5-z12, sempre apresentando o índice 2, como podemos ver nas tabelas.

Pentacordes relacionados

\begin{tabular}{|c|c|}
\hline Conjunto & Vetor intervalar \\
\hline $5-\mathrm{z} 37$ & {$[212320]$} \\
\hline $5-\mathrm{z} 17$ & {$[212320]$} \\
\hline $5-15$ & {$[220222]$} \\
\hline $5-22$ & {$[202321]$} \\
\hline $5-\mathrm{z} 12$ & {$[222121]$} \\
\hline
\end{tabular}


Tetracordes da região central

\begin{tabular}{|c|c|}
\hline Conjunto & Vetor intervalar \\
\hline $4-18$ & {$[102111]$} \\
\hline $4-17$ & {$[102210]$} \\
\hline $4-19$ & {$[101310]$} \\
\hline $4-11$ & {$[121110]$} \\
\hline $4-13$ & {$[112011]$} \\
\hline $4-10$ & {$[122010]$} \\
\hline
\end{tabular}

Conjuntos da região superior

\begin{tabular}{|c|c|}
\hline Conjunto & Vetor intervalar \\
\hline $4-5$ & {$[210111]$} \\
\hline $4-16$ & {$[110121]$} \\
\hline $4-8$ & {$[200121]$} \\
\hline $4-9$ & {$[200022]$} \\
\hline $5-15$ & {$[220222]$} \\
\hline $4-6$ & {$[210021]$} \\
\hline $3-5$ & {$[100011]$} \\
\hline $3-4$ & {$[100110]$} \\
\hline
\end{tabular}

Tab. 4: Análise de invariâncias entre vetores intervalares dos conjuntos da Região Central e Superior

Analisamos estes dados e propomos a construção de uma rede de conjuntos conexos por maior parcimônia entre vetores intervalares (Fig.4), relacionando todos os tetracordes da rede de projeções por inversão com pelo menos quatro entradas de vetores em comum. Percebemos que os tetracordes mantiveram seus posicionamentos referentes ao sistema original, o que demostra que o procedimento de projeção por inversão, além de prever a reiteração das alturas selecionadas como subconjuntos, também suscita a invariância de entradas de vetores intervalares entre os conjuntos. Apontamos na figura também a distribuição proporcional entre tetracordes simétricos $|\mathrm{s}|$ e assimétricos $|\mathrm{a}|$. 


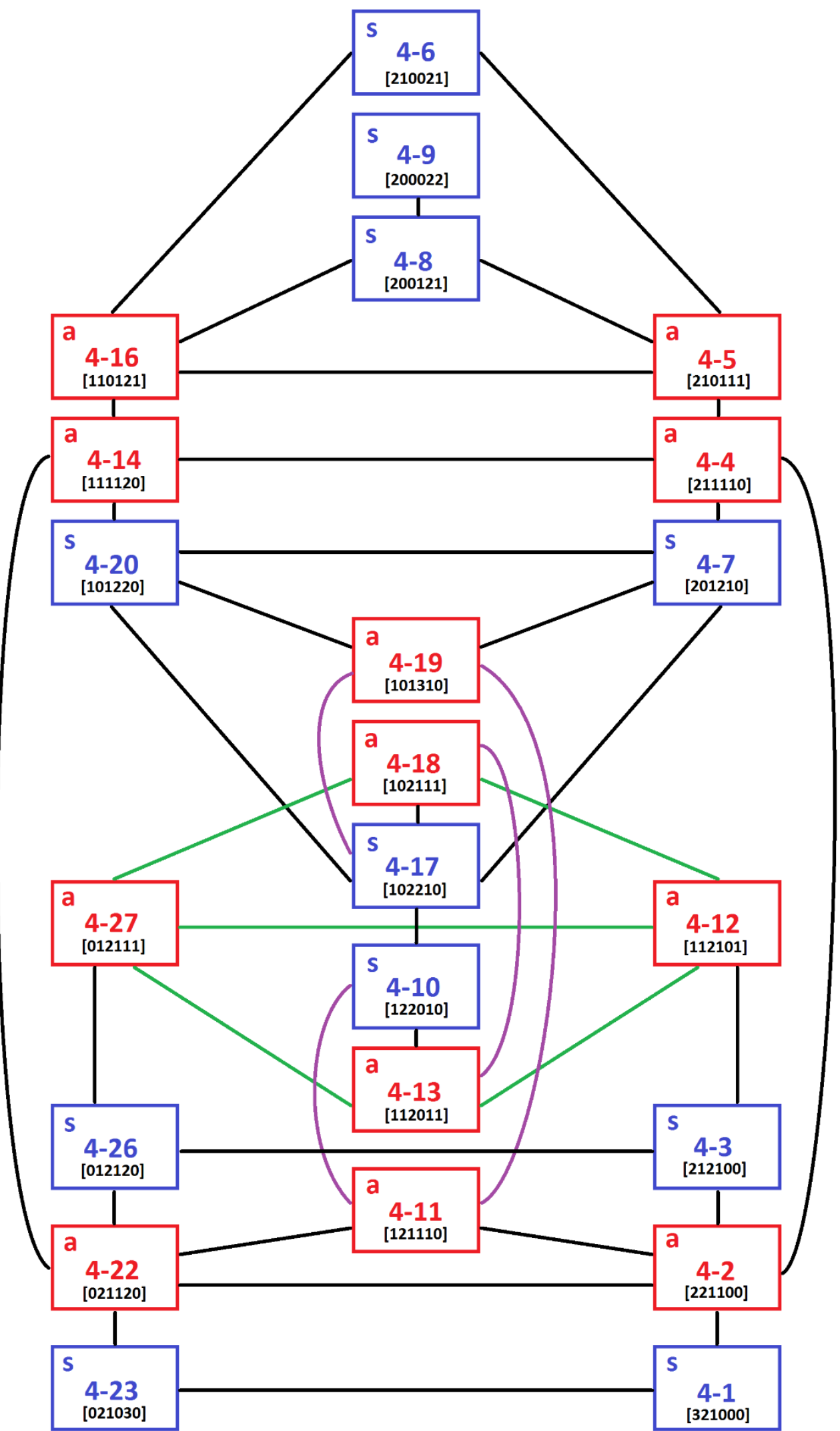

Fig. 4: Rede de tetracordes relacionados por um maior número de entradas de vetores intervalares invariantes

Além disso, destacamos que esta estrutura de conjuntos relacionados por invariância de vetores também exibe uma organização simétrica, apresentando uma distri- 
buição equivalente de seus tetracordes, reiterando as regiões $C 5$, C1, central e superior (Fig.5 e Fig.6).
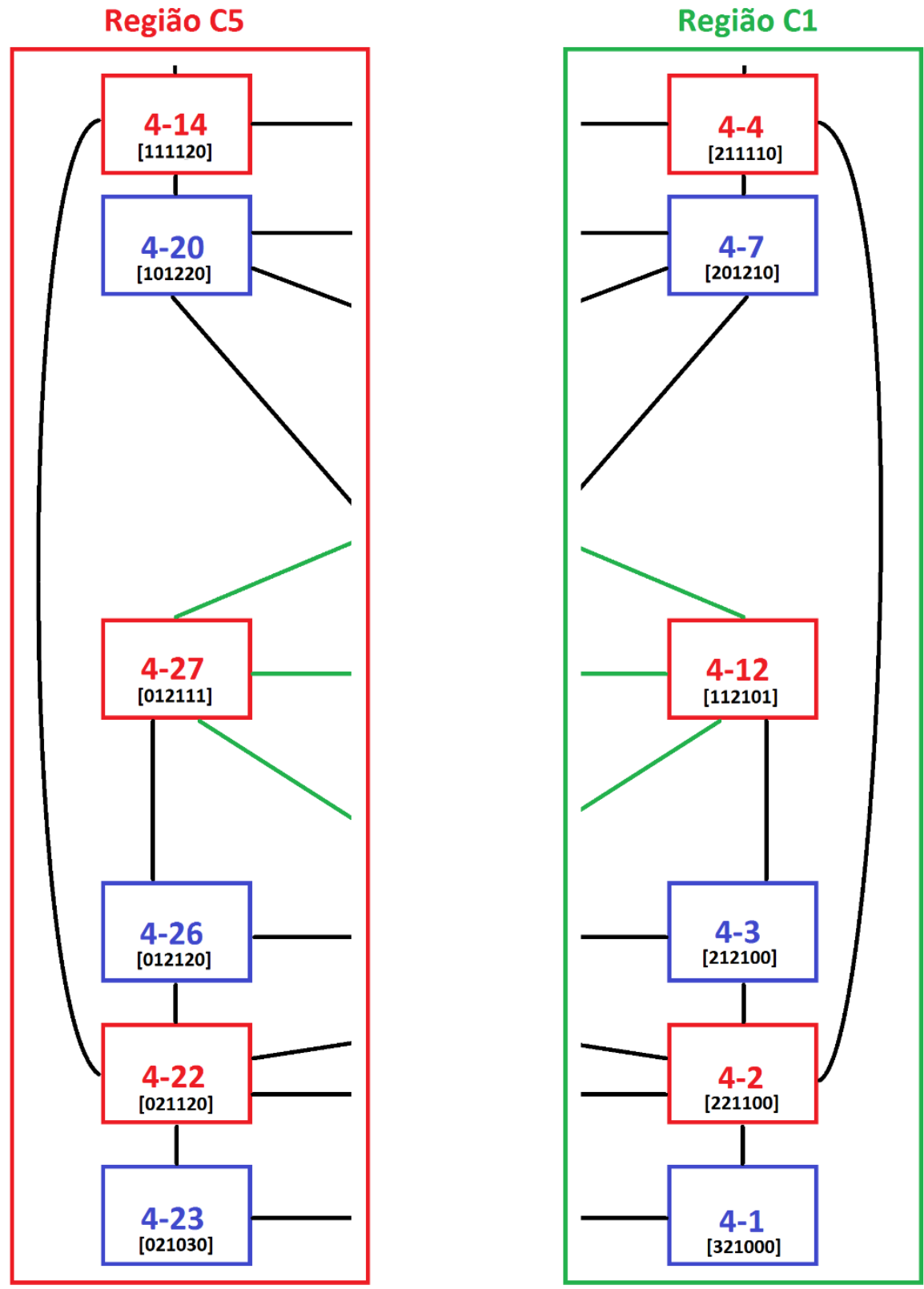

Fig. 5: Rede de tetracordes relacionados por vetores intervalares invariantes, reiteração das regiões C1 e C5 


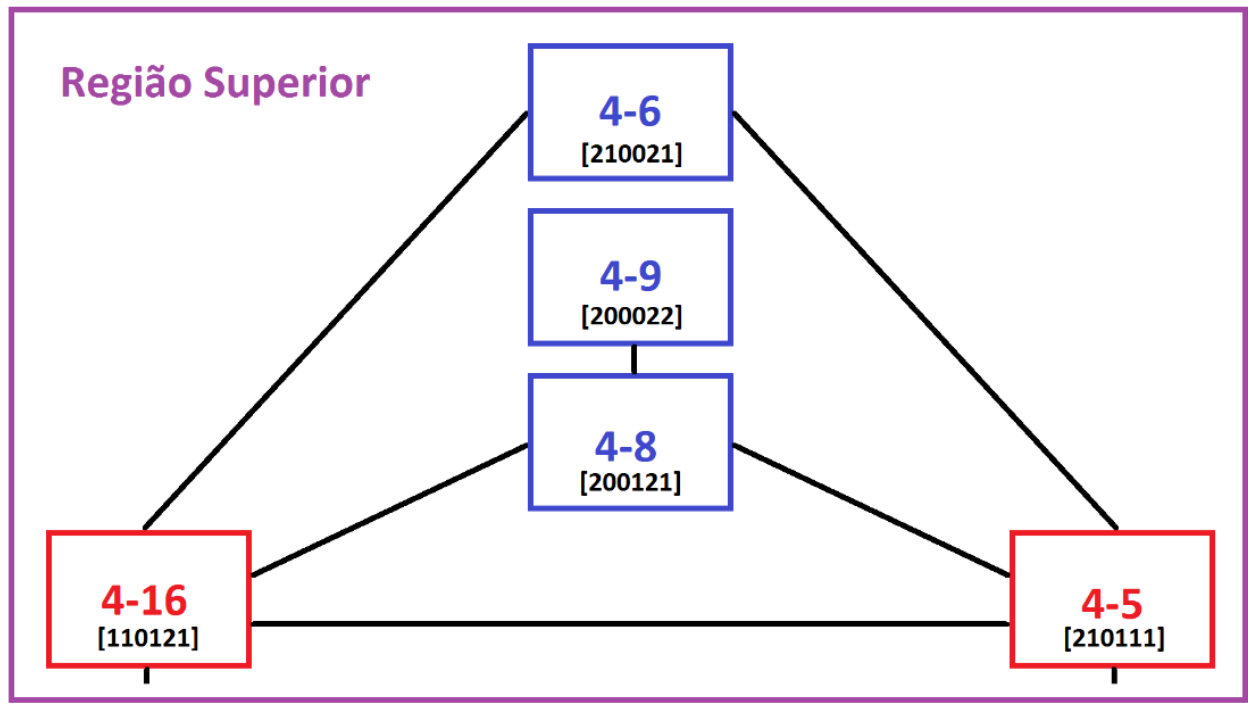

Região Central

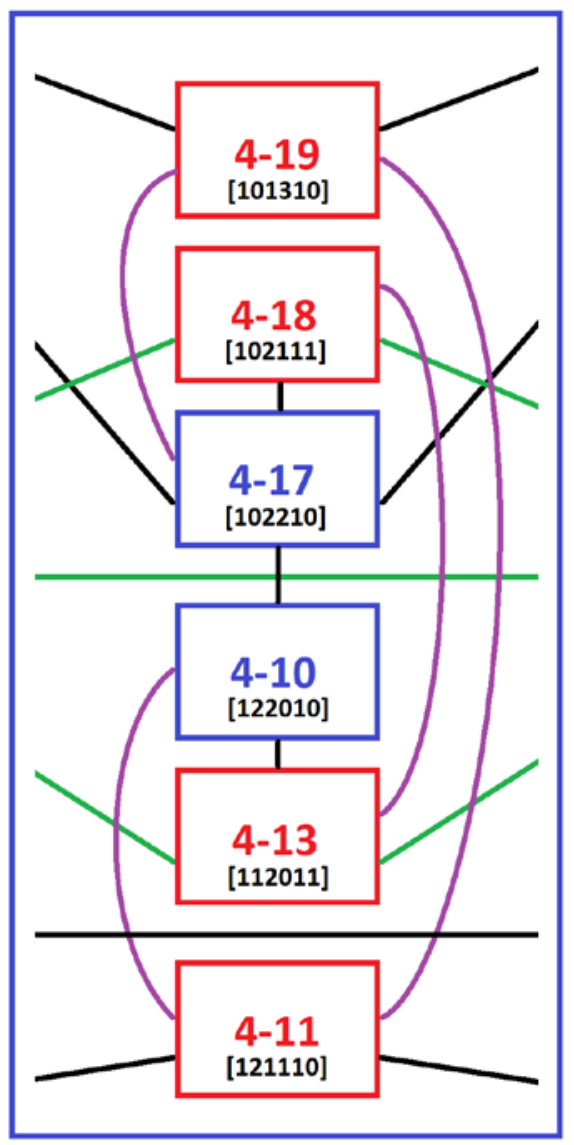

Fig. 6: Rede de tetracordes relacionados por vetores intervalares invariantes, reiteração das regiões Central e Superior 


\section{Perspectiva a partir da "constelação Morris"}

Em nossa investigação sobre as propriedades da rede de projeções por inversão, percebemos que esta estrutura provavelmente pode ser expandida até a abrangência de uma extensa quantidade de conjuntos de alturas existentes no sistema de doze sons, incluídos em um mesmo sistema transversal e simétrico. Ou seja, que o que mostramos anteriormente foram dois recortes deste sistema mais amplo ainda não explorado, construídos a partir de diferentes perspectivas, escolhendo alguns grupos de alturas em particular como pontos de partida para as projeções por inversão. A princípio trabalhamos a partir da rede de alturas (Tonnetz) com tricordes (Mod3), construindo uma rede de conjuntos relacionados por projeções a partir de díades implícitas nestes grupos de alturas (vértices dos triângulos que compõem um Tonnetz). Na sequência ampliamos este primeiro sistema apresentado, incluindo também conjuntos relacionados a partir de projeções a partir de alturas individuais dos tricordes (arestas dos triângulos que compõem um Tonnetz).

Verificamos que as duas versões da rede de projeções por inversão reiteram uma disposição proporcional entre grupos relacionados por multiplicação M5 e M7, opondo os diversos conjuntos de alturas em regiões $\mathrm{C} 1$ e C5, intermediadas por uma região central e outra superior que se posicionam como uma mediatriz de todo o sistema. Esta constatação corrobora nossa hipótese de que a simetria intervalar possa ser uma propriedade inerente ao próprio sistema cromático, aparecendo como característica invariante em diversas facetas do uso das doze alturas, valendo para ambos os universos tonal e pós-tonal.

Na sequência iremos apresentar outra perspectiva mais extensa para a rede de projeções por inversão, explorando agora as possibilidades de projeções por inversão a partir dos tetracordes que compõem a "constelação Morris" (SALLMEN, 2011) - os conjuntos 4-12, 4-13, 4-18 e 4-27 - juntos aos tetracordes 4-z15 e 4z29. Mostraremos assim que as características simétricas deste sistema e a correlação por multiplicação pelos índices M5 e $M 7$ são reiterados em todos os níveis de abrangência da rede de projeções por inversão, seja qual for a perspectiva e a dimensão do recorte que selecionarmos.

\subsection{Projeções de hexacordes simétricos}

Agora aplicaremos a matriz de soma (Tab.5) para a tétrade de Dó Maior com sétima menor (conjunto 4-27, numeração correspondente também para acordes meio diminutos), uma estrutura que não permite que seu grupo completo de quatro alturas (Mod4) se alinhe em pares em torno de um mesmo eixo de soma (assimétrico em sua totalidade), mas que apresenta subconjuntos simétricos (um tricorde (Mod3), seis pares de alturas (Mod2) e uma nota isolada (Mod1)) orientados por eixos distintos (Tab.6). 


\begin{tabular}{|c|c|c|c|c|}
\hline$(+)$ & Dó (0) & Mi (4) & Sol (7) & Sib (10) \\
\hline Dó (0) & 0 & 4 & 7 & 10 \\
\hline Mi (4) & 4 & 8 & 11 & 2 \\
\hline Sol (7) & 7 & 11 & 2 & 5 \\
\hline Sib (10) & 10 & 2 & 5 & 8 \\
\hline
\end{tabular}

Tab. 5: Matriz de soma para o acorde Do Maior com Sétima

\begin{tabular}{c|ccc|}
\multicolumn{2}{c|}{ Soma } & Pares & Conjunto \\
\hline 0 & $0 / 0$ & & $1-1$ \\
1 & - & $4 / 10$ & - \\
2 & $7 / 7$ & & $3-10$ \\
3 & - & & - \\
4 & $0 / 4$ & & $2-4$ \\
5 & $7 / 10$ & & $2-3$ \\
6 & - & $10 / 10$ & - \\
7 & $0 / 7$ & & $2-5$ \\
8 & $4 / 4$ & & $2-6$ \\
9 & - & & - \\
10 & $0 / 10$ & & $2-2$ \\
11 & $4 / 7$ & & $2-3$ \\
\hline
\end{tabular}

Tab. 6: Subconjuntos simétricos implícitos no acorde Dó Maior com Sétima e respectivos eixos

Uma forma de transformar este acorde Maior 7 (conjunto 4-27), originalmente assimétrico em sua totalidade, em um conjunto integralmente simétrico, é escolher um par de alturas como subconjunto, identificar o eixo de soma inerente entre estas duas notas e agregar duas alturas adicionais junto a terceira e quarta nota do tetracorde, correspondendo respectivamente à mesma soma do eixo em questão, gerando assim um hexacorde simétrico. Para obter estas alturas adicionais, basta subtrair o valor de soma do eixo pelo valor da altura sem par e teremos a nota compatível que completa a simetria do conjunto como um todo. Reiteramos assim a seguinte fórmula:

Sendo:

\begin{tabular}{|l|}
\hline \multicolumn{1}{|c|}{ Altura $|\mathbf{x}|+$ Altura $|\mathbf{y}|=$ Soma $|\mathbf{s}|(\operatorname{Mod} 12)$} \\
\hline Então: \\
\hline
\end{tabular}


Considerando que o acorde Maior 7 (conjunto 4-27 (Mod4)) tem seis pares de notas como subconjuntos simétricos (Mod2), cada qual orientado pelo seu respectivo eixo, poderemos estipular e adicionar uma nota correspondente para cada uma das duas alturas isoladas nos seis casos, reiterando o mesmo valor de soma do respectivo eixo, projetando assim cinco possibilidades de hexacordes (Mod6) e um pentacorde (Mod5) totalmente simétricos (devido a reiteração de uma nota), originários da mesma tétrade referida: os conjuntos 6-z23, 6-z29, 6-z42, 6-z49, 6-z50 e 5-34 (Tab.7).

Mapeamos nas tabelas abaixo (Tab.7 e Tab.8) cada uma destas possibilidades de projeções, observando na sequência: o conjunto referido, a altura escolhida como subconjunto (Mod2), a soma $|s|$ gerada em torno deste subconjunto, as demais alturas $|x|$ do tetracorde e seus respectivos pares $|y|$ de mesma soma do eixo invariante e o conjunto do hexacorde projetado (pentacorde, em um dos casos).

\begin{tabular}{|c|c|c|c|c|}
\hline $\begin{array}{c}\text { Subconjunto } \\
(\operatorname{Mod} 2)\end{array}$ & Soma $|\mathbf{s}|$ & Altura $|\mathrm{x}|$ & $\begin{array}{l}\text { Altura }|\mathrm{y}| \\
|\mathrm{y}|=|\mathbf{s}|-|\mathrm{x}|\end{array}$ & Projeção \\
\hline \multirow[t]{2}{*}[0,4]{} & \multirow[t]{2}{*}{4} & 7 & 9 & {$[4,6,7,9,10,0]$} \\
\hline & & 10 & 6 & $6-z 23$ \\
\hline \multirow[t]{2}{*}[0,7]{} & \multirow[t]{2}{*}{7} & 4 & 3 & {$[3,4,7,9,10,0]$} \\
\hline & & 10 & 9 & 6-z50 \\
\hline \multirow[t]{2}{*}[0,10]{} & \multirow[t]{2}{*}{10} & 4 & 6 & {$[3,4,6,7,10,0]$} \\
\hline & & 7 & 3 & 6-z49 \\
\hline \multirow[t]{2}{*}[4,7]{} & \multirow[t]{2}{*}{11} & 0 & 11 & {$[10,11,0,1,4,7]$} \\
\hline & & 10 & 1 & $6-z 42$ \\
\hline \multirow[t]{2}{*}[4,10]{} & \multirow[t]{2}{*}{2} & 0 & 2 & {$[10,0,2,4,7]$} \\
\hline & & 7 & 7 & $5-34$ \\
\hline \multirow[t]{2}{*}[7,10]{} & \multirow[t]{2}{*}{5} & 0 & 5 & {$[10,0,1,4,5,7]$} \\
\hline & & 4 & 1 & $6-z 29$ \\
\hline
\end{tabular}

Tab. 7: Possibilidades de projeções a partir de um acorde Maior com Sétima (conjunto 4-27)

Selecionamos outros três tetracordes para mapearmos outras possibilidades de projeções (Tab.8), utilizando os mesmos critérios aplicados acima - os conjuntos 4-12, 4-13 e 4-18 - os quais juntos ao conjunto 4-27 formam a "constelação Morris" (Fig.7, SALLMEN, 2011). 
Example 1. The MORRIS Constellation (after Soderberg 1998)

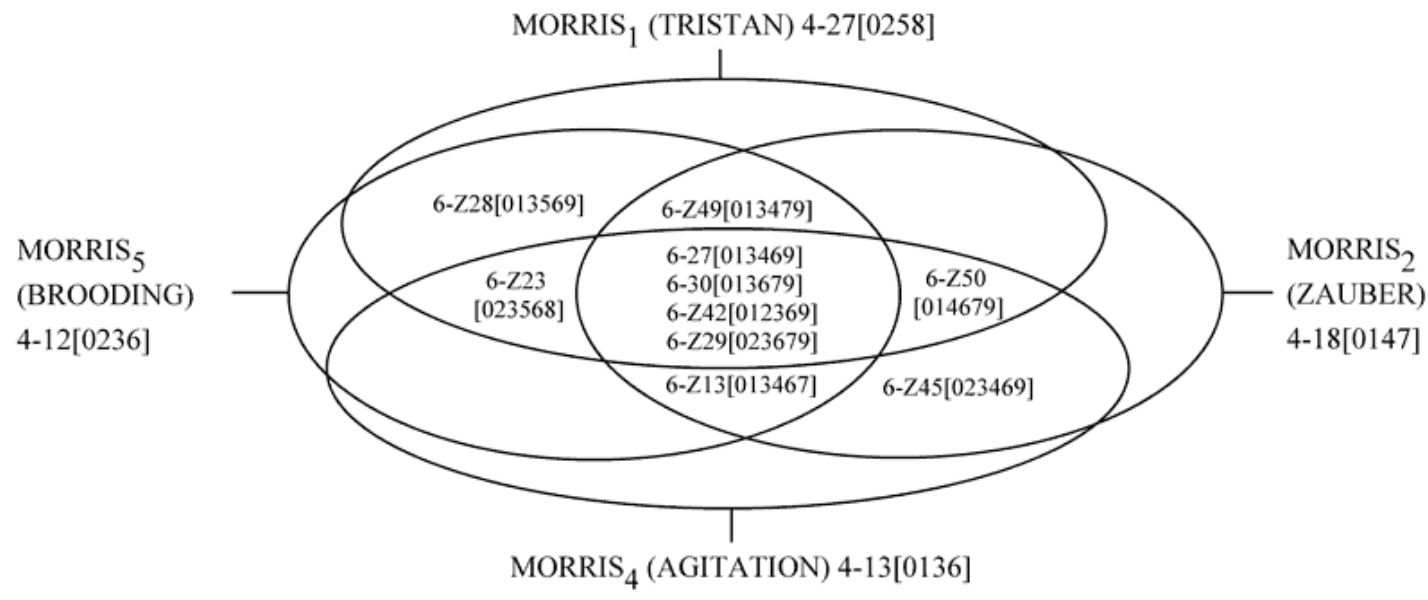

Fig. 7: Correspondência com a "constelação Morris" (SALLMEN, 2011)

A seguir apresentamos esses dados levantados dispostos nas tabelas.

Conjunto 4-12, alturas $[0,2,3,6]$

\begin{tabular}{|c|c|c|c|c|}
\hline $\begin{array}{c}\text { Subconjunto } \\
(\operatorname{Mod} 2)\end{array}$ & Soma $|s|$ & Altura $|\mathbf{x}|$ & $\begin{array}{l}\text { Altura }|y| \\
|y|=|\mathbf{s}|-|x|\end{array}$ & Projeção \\
\hline \multirow[t]{2}{*}[0,2]{} & \multirow[t]{2}{*}{2} & 3 & 11 & {$[11,0,2,3,6,8]$} \\
\hline & & 6 & 8 & $6-z 49$ \\
\hline \multirow[t]{2}{*}[0,3]{} & \multirow[t]{2}{*}{3} & 2 & 1 & {$[0,1,2,3,6,9]$} \\
\hline & & 6 & 9 & $6-z 42$ \\
\hline \multirow[t]{2}{*}[0,6]{} & \multirow[t]{2}{*}{6} & 2 & 4 & {$[0,2,3,4,6]$} \\
\hline & & 3 & 3 & $5-8$ \\
\hline \multirow[t]{2}{*}[2,3]{} & \multirow[t]{2}{*}{5} & 0 & 5 & {$[11,0,2,3,5,6]$} \\
\hline & & 6 & 11 & 6-z13 \\
\hline \multirow[t]{2}{*}[2,6]{} & \multirow[t]{2}{*}{8} & 0 & 8 & {$[0,2,3,5,6,8]$} \\
\hline & & 3 & 5 & $6-z 23$ \\
\hline \multirow[t]{2}{*}[3,6]{} & \multirow[t]{2}{*}{9} & 0 & 9 & {$[0,2,3,6,7,9]$} \\
\hline & & 2 & 7 & $6-z 29$ \\
\hline
\end{tabular}


Conjunto 4-13, alturas $[0,1,3,6]$

\begin{tabular}{|c|c|c|c|c|}
\hline $\begin{array}{c}\text { Subconjunto } \\
\text { (Mod2) }\end{array}$ & Soma $|\mathbf{s}|$ & Altura $|\mathrm{x}|$ & $\begin{array}{l}\text { Altura }|y| \\
|y|=|s|-|x|\end{array}$ & Projeção \\
\hline \multirow[t]{2}{*}[0,1]{} & \multirow[t]{2}{*}{1} & 3 & 10 & {$[6,7,10,0,1,3]$} \\
\hline & & 6 & 7 & $6-z 50$ \\
\hline \multirow[t]{2}{*}[0,3]{} & \multirow[t]{2}{*}{3} & 1 & 2 & {$[0,1,2,3,6,9]$} \\
\hline & & 6 & 9 & $6-z 42$ \\
\hline \multirow[t]{2}{*}[0,6]{} & \multirow[t]{2}{*}{6} & 1 & 5 & {$[0,1,3,5,6]$} \\
\hline & & 3 & 3 & $5-z 12$ \\
\hline \multirow[t]{2}{*}[1,3]{} & \multirow[t]{2}{*}{4} & 0 & 4 & {$[10,0,1,3,4,6]$} \\
\hline & & 6 & 10 & $6-z 23$ \\
\hline \multirow[t]{2}{*}[1,6]{} & \multirow[t]{2}{*}{7} & 0 & 7 & {$[0,1,3,4,6,7]$} \\
\hline & & 3 & 4 & 6-z13 \\
\hline \multirow[t]{2}{*}[3,6]{} & \multirow[t]{2}{*}{9} & 0 & 9 & {$[6,8,9,0,1,3]$} \\
\hline & & 1 & 8 & $6-z 29$ \\
\hline
\end{tabular}

\section{Conjunto 4-18, alturas $[0,1,4,7]$}

\begin{tabular}{|c|c|c|c|c|}
\hline $\begin{array}{c}\text { Subconjunto } \\
(\operatorname{Mod} 2)\end{array}$ & Soma $|\mathbf{s}|$ & Altura $|\mathrm{x}|$ & $\begin{array}{l}\text { Altura }|\mathbf{y}| \\
|\mathbf{y}|=|\mathbf{S}|-|\mathbf{x}|\end{array}$ & Projeção \\
\hline \multirow[t]{2}{*}[0,1]{} & \multirow[t]{2}{*}{1} & 4 & 9 & {$[0,1,4,6,7,9]$} \\
\hline & & 7 & 6 & $6-z 50$ \\
\hline \multirow[t]{2}{*}[0,4]{} & \multirow[t]{2}{*}{4} & 1 & 3 & {$[0,1,3,4,7,9]$} \\
\hline & & 7 & 9 & $6-z 49$ \\
\hline \multirow[t]{2}{*}[0,7]{} & \multirow[t]{2}{*}{7} & 1 & 6 & {$[0,1,3,4,6,7]$} \\
\hline & & 4 & 3 & 6-z13 \\
\hline \multirow[t]{2}{*}[1,4]{} & \multirow[t]{2}{*}{5} & 0 & 5 & {$[10,0,1,4,5,7]$} \\
\hline & & 7 & 10 & $6-z 29$ \\
\hline \multirow[t]{2}{*}[1,7]{} & \multirow[t]{2}{*}{8} & 0 & 8 & {$[0,1,4,7,8]$} \\
\hline & & 4 & 4 & $5-22$ \\
\hline \multirow[t]{2}{*}[4,7]{} & \multirow[t]{2}{*}{11} & 0 & 11 & {$[10,11,0,1,4,7]$} \\
\hline & & 1 & 10 & $6-z 42$ \\
\hline
\end{tabular}

Tab. 8: Mapeamento de projeções a partir dos conjuntos 4-12, 4-13 e 4-18 
A partir destas informações, podemos propor um novo sistema de conjuntos relacionados por projeções a partir de inversões. Apresentamos a seguir um gráfico (Fig.8) relacionando os tetracordes 4-27, 4-12, 4-13 e 4-18 aos hexacordes 6-z13, 6-z23, 6-z29, $6-z 42,6-z 45$ e $6-z 50$. Temos ainda a presença de quatro pentacordes - 5-8, 5-z12, 5-22 e 5-34 - que aparecem por reiterações de alturas no processo de projeção de alguns tetracordes.

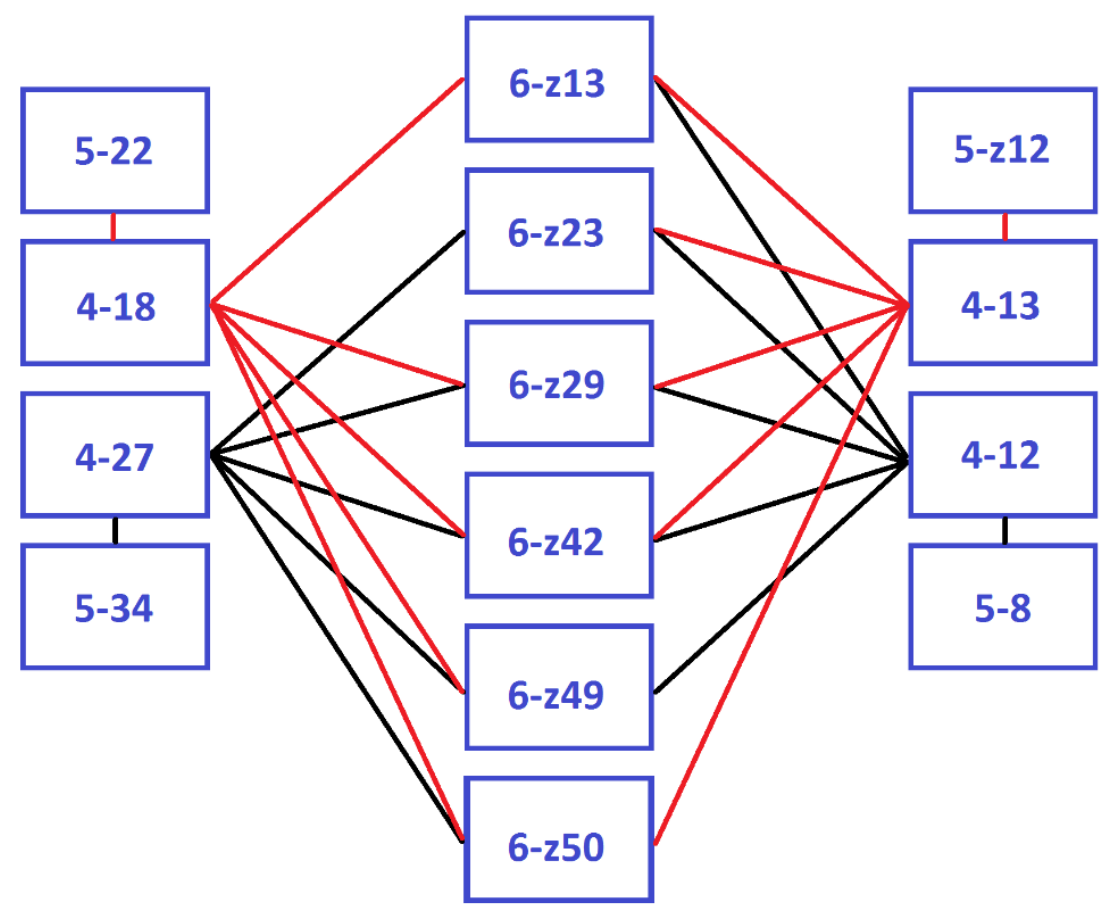

Fig. 8: Sistema de conjuntos relacionados por projeções a partir de inversões em torno dos tetracordes 4-27, 4-12, 4-13 e 4-18

Também nesta relação entre hexacordes e pentacordes projetados por inversão a partir de tetracordes temos a reiteração de entradas de vetores intervalares (Tab.9). Todos os hexacordes têm em comum o índice 4 na entrada três (terças menores e sextas maiores) e índice 2 na entrada seis (trítonos), além de se relacionarem em pares mantendo quatro entradas de vetores em comum e dois invertidos entre si (sempre entre os índices 2 e 3), exceto os conjuntos Z-relacionados com todos os vetores intervalares em comum (6-z13/6-z42 e 6-z29/6-z50). O mesmo acontece na relação entre os tetracordes: têm em comum o índice 2 na entrada três (terças menores e sextas maiores) e índice 1 na entrada seis (trítonos), além de também se relacionarem em pares mantendo quatro entradas de vetores em comum e dois invertidos entre si (sempre entre os índices 0 e 1). 


\begin{tabular}{|c|c|c|c|}
\hline Hexacorde & Vetores & Tetracorde & Vetores \\
\hline $6-z 13$ & {$[324222]$} & $4-12$ & {$[112101]$} \\
\hline $6-z 23$ & {$[234222]$} & $4-27$ & {$[012111]$} \\
\hline $6-z 29$ & {$[224232]$} & $4-18$ & {$[102111]$} \\
\hline $6-z 42$ & {$[324222]$} & $4-13$ & {$[112011]$} \\
\hline $6-z 49$ & {$[224322]$} & & \\
\cline { 1 - 2 } $6-\mathrm{z} 50$ & {$[224232]$} & & \\
\hline
\end{tabular}

\begin{tabular}{|c|c|c|c|}
\hline Hexacordes & Vetores & Hexacordes & Vetores \\
\hline $6-z 13$ & & $6-z 13$ & [324222] \\
\hline $6-z 23$ & [234222] & $6-z 29$ & [224232] \\
\hline $6-z 13$ & {$[324222]$} & $6-z 13$ & [324222] \\
\hline $6-z 42$ & [324222] & $6-z 49$ & [224322] \\
\hline $6-z 13$ & [324222] & $6-z 23$ & [234222] \\
\hline $6-z 50$ & [224232] & $6-z 29$ & [224232] \\
\hline $6-z 23$ & [234222] & $6-z 23$ & [234222] \\
\hline $6-z 42$ & [324222] & $6-z 49$ & [224322] \\
\hline $6-z 23$ & {$[234222]$} & $6-z 29$ & [224232] \\
\hline $6-z 50$ & [224232] & $6-z 42$ & [324222] \\
\hline $6-z 29$ & [224232] & $6-z 29$ & [224232] \\
\hline $6-z 49$ & [224322] & $6-z 50$ & [224232] \\
\hline $6-z 42$ & {$[324222]$} & $6-z 42$ & [324222] \\
\hline $6-z 49$ & [224322] & $6-z 50$ & [224232] \\
\hline $6-z 49$ & [224322] & & \\
\hline $6-z 50$ & [224232] & & \\
\hline
\end{tabular}




\begin{tabular}{|c|c|c|c|}
\hline Tetracordes & Vetores & Tetracordes & Vetores \\
\hline $4-12$ & {$[112101]$} & $4-12$ & {$[112101]$} \\
$4-27$ & {$[012111]$} & $4-18$ & {$[102111]$} \\
\hline $4-12$ & {$[112101]$} & $4-27$ & {$[01211]$} \\
$4-13$ & {$[112011]$} & $4-18$ & {$[10211]$} \\
\hline $4-27$ & {$[012111]$} & $4-18$ & {$[10211]$} \\
$4-13$ & {$[112011]$} & $4-13$ & {$[112011]$} \\
\hline
\end{tabular}

Tab. 9: Invariâncias entre vetores intervalares

Podemos verificar ainda que quatro destes hexacordes se relacionam por multiplicação M5 ou M7 entre si, dispostos em dois grupos correspondentes às regiões $\mathrm{C} 1$ e C5 (de acordo com o maior índice nas entradas um e cinco dos vetores intervalares), como podemos ver na análise apresentada na tabela abaixo (Tab.10). Interessante que, por serem ambos pares de conjuntos Z-relacionados, temos o mesmo parâmetro de multiplicação atribuído entre os quatro hexacordes.

\begin{tabular}{|c|c|c|c|}
\hline Região C5 & Vetores & Região C1 & Vetores \\
\hline $6-z 50$ & {$[224232]$} & $6-z 13$ & {$[324222]$} \\
\hline $6-z 29$ & {$[224232]$} & $6-z 42$ & {$[324222]$} \\
\hline $6-z 29$ & {$[224232]$} & $6-z 13$ & {$[324222]$} \\
\hline $6-z 50$ & {$[224232]$} & $6-z 42$ & {$[324222]$} \\
\hline
\end{tabular}

Tab. 10: Relações por multiplicação pelos fatores M5 e M7 entre hexacordes Z-relacionados

Outro par de tetracordes que se relaciona com parte deste grupo de hexacordes são os conjuntos Z-relacionados 4-z15 e 4-z29, que podem projetar ambos os conjuntos 6-z13, 6-z23, 6-z49 e 6-z50, além de outros dois hexacordes cada: 6-z38 e 6-z26 para 4-z29 e 6-z6 e 6-z4 para 4-z15. Estas possibilidades de projeções foram mapeadas e apresentadas nas tabelas abaixo (Tab.11): 
Conjunto 4-z15, alturas $[0,1,4,6]$

\begin{tabular}{|c|c|c|c|c|}
\hline $\begin{array}{c}\text { Subconjunto } \\
\text { (Mod2) }\end{array}$ & Soma $|s|$ & Altura $|\mathbf{x}|$ & $\begin{array}{l}\text { Altura }|\mathrm{y}| \\
|\mathrm{y}|=|\mathbf{s}|-|\mathrm{x}|\end{array}$ & Projeção \\
\hline \multirow[t]{2}{*}[0,1]{} & \multirow[t]{2}{*}{1} & 4 & 9 & {$[0,1,4,6,7,9]$} \\
\hline & & 6 & 7 & $6-z 50$ \\
\hline \multirow[t]{2}{*}[0,4]{} & \multirow[t]{2}{*}{4} & 1 & 3 & {$[10,0,1,3,4,6]$} \\
\hline & & 6 & 10 & $6-z 23$ \\
\hline \multirow[t]{2}{*}[0,6]{} & \multirow[t]{2}{*}{6} & 1 & 5 & {$[0,1,2,4,5,6]$} \\
\hline & & 4 & 2 & $6-z 4$ \\
\hline \multirow[t]{2}{*}[1,4]{} & \multirow[t]{2}{*}{5} & 0 & 5 & {$[11,0,1,4,5,6]$} \\
\hline & & 6 & 11 & $6-z 6$ \\
\hline \multirow[t]{2}{*}[1,6]{} & \multirow[t]{2}{*}{7} & 0 & 7 & {$[0,1,3,4,6,7]$} \\
\hline & & 4 & 3 & 6-z13 \\
\hline \multirow[t]{2}{*}[4,6]{} & \multirow[t]{2}{*}{10} & 0 & 10 & {$[9,10,0,1,4,6]$} \\
\hline & & 1 & 9 & 6-z49 \\
\hline
\end{tabular}

Conjunto 4-z29, alturas $[0,1,3,7]$

\begin{tabular}{|c|c|c|c|c|}
\hline $\begin{array}{c}\text { Subconjunto } \\
\text { (Mod2) }\end{array}$ & Soma $|s|$ & Altura $|\mathbf{x}|$ & $\begin{array}{l}\text { Altura }|y| \\
|y|=|\mathbf{s}|-|x|\end{array}$ & Projeção \\
\hline \multirow[t]{2}{*}[0,1]{} & \multirow[t]{2}{*}{1} & 3 & 10 & {$[6,7,10,0,1,3]$} \\
\hline & & 7 & 6 & $6-z 50$ \\
\hline \multirow[t]{2}{*}[0,3]{} & \multirow[t]{2}{*}{3} & 1 & 2 & {$[0,1,2,3,7,8]$} \\
\hline & & 7 & 8 & $6-z 38$ \\
\hline \multirow[t]{2}{*}[0,7]{} & \multirow[t]{2}{*}{7} & 1 & 6 & {$[0,1,3,4,6,7]$} \\
\hline & & 3 & 4 & $6-z 13$ \\
\hline \multirow[t]{2}{*}[1,3]{} & \multirow[t]{2}{*}{4} & 0 & 4 & {$[0,1,3,4,7,9]$} \\
\hline & & 7 & 9 & $6-z 49$ \\
\hline \multirow[t]{2}{*}[1,7]{} & \multirow[t]{2}{*}{8} & 0 & 8 & {$[0,1,3,5,7,8]$} \\
\hline & & 3 & 5 & $6-\mathrm{z} 26$ \\
\hline \multirow[t]{2}{*}[3,7]{} & \multirow[t]{2}{*}{10} & 0 & 10 & {$[7,9,10,0,1,3]$} \\
\hline & & 1 & 9 & $6-z 23$ \\
\hline
\end{tabular}

Tab. 11: Possibilidades de projeções a partir dos conjuntos Z-relacionados 4-z15 e 4-z29

Outro caminho para observarmos a relação entre projeções de conjuntos por inversão é a partir do mapeamento dos tetracordes que podem gerar estes hexacordes 
simétricos a partir de eixos implícitos em subconjuntos de pares de alturas. Cada hexacorde tem três pares de alturas alinhados em torno do mesmo eixo, sendo que cada par projeta o hexacorde a partir de duas maneiras diferentes de dispor as demais quatro notas, assim organizadas em seis pares de tetracordes implícitos que se invertem entre si em torno do eixo invariante e, quando concatenados, projetam o mesmo hexacorde. Listamos a seguir os seis conjuntos de tetracordes que geram cada um dos dez hexacordes que relacionam os tetracordes 4-27, 4-12, 4-13, 4-18, 4-z15 e 4-z29 (Tab.12):

Conjunto 6-z29, (023679)

Alturas $[0,2,3,6,7,9]$

Eixo soma 9, pares $[0,9][2,7][3,6]$

\begin{tabular}{|c|c|c|c|c|c|}
\hline $\begin{array}{c}\text { Pares } \\
(\operatorname{Mod} 2)\end{array}$ & Soma $|\mathbf{s}|$ & Altura $|\mathbf{x}|$ & $\begin{array}{l}\text { Altura }|\mathrm{y}| \\
|\mathrm{y}|=|\mathbf{s}|-|\mathrm{x}|\end{array}$ & Conjunto & Tetracordes \\
\hline \multirow[t]{2}{*}[0,9]{} & \multirow[t]{2}{*}{9} & 2 & 7 & \multirow[t]{2}{*}{$4-13$} & {$[9,0,2,3]$} \\
\hline & & 3 & 6 & & {$[6,7,9,0]$} \\
\hline \multirow[t]{2}{*}[0,9]{} & \multirow[t]{2}{*}{9} & 2 & 7 & \multirow[t]{2}{*}{$4-27$} & {$[6,9,0,2]$} \\
\hline & & 6 & 3 & & {$[7,9,0,3]$} \\
\hline \multirow[t]{2}{*}[2,7]{} & \multirow[t]{2}{*}{9} & 0 & 9 & \multirow[t]{2}{*}{$4-14$} & {$[0,2,3,7]$} \\
\hline & & 3 & 6 & & {$[2,6,7,9]$} \\
\hline \multirow[t]{2}{*}[2,7]{} & \multirow[t]{2}{*}{9} & 0 & 9 & \multirow[t]{2}{*}{$4-16$} & {$[0,2,6,7]$} \\
\hline & & 6 & 3 & & {$[2,3,7,9]$} \\
\hline \multirow[t]{2}{*}[3,6]{} & \multirow[t]{2}{*}{9} & 0 & 9 & \multirow[t]{2}{*}{$4-12$} & {$[0,2,3,6]$} \\
\hline & & 2 & 7 & & {$[3,6,7,9]$} \\
\hline \multirow[t]{2}{*}[3,6]{} & \multirow[t]{2}{*}{9} & 0 & 9 & \multirow[t]{2}{*}{$4-18$} & {$[0,3,6,7]$} \\
\hline & & 7 & 2 & & {$[2,3,6,9]$} \\
\hline
\end{tabular}


Conjunto 6-z42, (012369)

Alturas $[0,1,2,3,6,9]$

Eixo soma 3, pares $[1,2][0,3][6,9]$

\begin{tabular}{|c|c|c|c|c|c|}
\hline $\begin{array}{c}\text { Pares } \\
(\operatorname{Mod} 2)\end{array}$ & Soma $|\mathbf{s}|$ & Altura $|\mathrm{x}|$ & $\begin{array}{l}\text { Altura }|y| \\
|y|=|s|-|x|\end{array}$ & Conjunto & Tetracordes \\
\hline \multirow[t]{2}{*}[1,2]{} & \multirow[t]{2}{*}{3} & 0 & 3 & \multirow[t]{2}{*}{$4-5$} & {$[0,1,2,6]$} \\
\hline & & 6 & 9 & & {$[9,1,2,3]$} \\
\hline \multirow[t]{2}{*}[1,2]{} & \multirow[t]{2}{*}{3} & 0 & 3 & \multirow[t]{2}{*}{ 4-4 } & {$[9,0,1,2]$} \\
\hline & & 9 & 6 & & {$[1,2,3,6]$} \\
\hline \multirow[t]{2}{*}[0,3]{} & \multirow[t]{2}{*}{3} & 1 & 2 & \multirow[t]{2}{*}{$4-13$} & {$[0,1,3,6]$} \\
\hline & & 6 & 9 & & {$[9,0,2,3]$} \\
\hline \multirow[t]{2}{*}[0,3]{} & \multirow[t]{2}{*}{3} & 1 & 2 & \multirow[t]{2}{*}{$4-12$} & {$[9,0,1,3]$} \\
\hline & & 9 & 6 & & {$[0,2,3,6]$} \\
\hline \multirow[t]{2}{*}[6,9]{} & \multirow[t]{2}{*}{3} & 1 & 2 & \multirow[t]{2}{*}{$4-18$} & {$[6,9,0,1]$} \\
\hline & & 0 & 3 & & {$[2,3,6,9]$} \\
\hline \multirow[t]{2}{*}[6,9]{} & \multirow[t]{2}{*}{3} & 1 & 2 & \multirow[t]{2}{*}{$4-27$} & {$[1,3,6,9]$} \\
\hline & & 3 & 0 & & {$[6,9,0,2]$} \\
\hline
\end{tabular}

Conjunto 6-z23, (023568)

Alturas $[0,2,3,5,6,8]$

Eixo soma 8 , pares $[0,8][2,6][3,5]$

\begin{tabular}{|c|c|c|c|c|c|}
\hline $\begin{array}{c}\text { Pares } \\
(\operatorname{Mod} 2)\end{array}$ & Soma $|s|$ & Altura $|\mathrm{x}|$ & $\begin{array}{l}\text { Altura }|\mathrm{y}| \\
|\mathrm{y}|=|\mathrm{s}|-|\mathrm{x}|\end{array}$ & Conjunto & Tetracordes \\
\hline \multirow[t]{2}{*}[0,8]{} & \multirow[t]{2}{*}{8} & 2 & 6 & \multirow[t]{2}{*}{$4-z 29$} & {$[8,0,2,3]$} \\
\hline & & 3 & 5 & & {$[5,6,8,0]$} \\
\hline \multirow[t]{2}{*}[0,8]{} & \multirow[t]{2}{*}{8} & 2 & 6 & \multirow[t]{2}{*}{$4-27$} & {$[0,2,5,8]$} \\
\hline & & 5 & 3 & & {$[0,3,6,8]$} \\
\hline \multirow[t]{2}{*}[2,6]{} & \multirow[t]{2}{*}{8} & 0 & 8 & \multirow[t]{2}{*}{$4-12$} & {$[0,2,3,6]$} \\
\hline & & 3 & 5 & & {$[2,5,6,8]$} \\
\hline \multirow[t]{2}{*}[2,6]{} & \multirow[t]{2}{*}{8} & 0 & 8 & \multirow[t]{2}{*}{$4-z 15$} & {$[0,2,5,6]$} \\
\hline & & 5 & 3 & & {$[2,3,6,8]$} \\
\hline \multirow[t]{2}{*}[3,5]{} & \multirow[t]{2}{*}{8} & 0 & 8 & \multirow[t]{2}{*}{$4-10$} & {$[0,2,3,5]$} \\
\hline & & 2 & 6 & & {$[3,5,6,8]$} \\
\hline \multirow[t]{2}{*}[3,5]{} & \multirow[t]{2}{*}{8} & 0 & 8 & \multirow[t]{2}{*}{$4-13$} & {$[0,3,5,6]$} \\
\hline & & 6 & 2 & & {$[2,3,5,8]$} \\
\hline
\end{tabular}


Conjunto 6-z50, (014679)

Alturas $[0,1,4,6,7,9]$

Eixo soma 1, pares $[0,1][4,9][6,7]$

\begin{tabular}{|c|c|c|c|c|c|}
\hline $\begin{array}{c}\text { Pares } \\
(\operatorname{Mod} 2)\end{array}$ & Soma $|\mathrm{s}|$ & Altura $|\mathrm{x}|$ & $\begin{array}{l}\text { Altura }|y| \\
|y|=|s|-|x|\end{array}$ & Conjunto & Tetracordes \\
\hline \multirow[t]{2}{*}[0,1]{} & \multirow[t]{2}{*}{1} & 4 & 9 & \multirow[t]{2}{*}{$4-z 15$} & {$[0,1,4,6]$} \\
\hline & & 6 & 7 & & {$[7,9,0,1]$} \\
\hline \multirow[t]{2}{*}[0,1]{} & \multirow[t]{2}{*}{1} & 4 & 9 & \multirow[t]{2}{*}{$4-18$} & {$[0,1,4,7]$} \\
\hline & & 7 & 6 & & {$[6,9,0,1]$} \\
\hline \multirow[t]{2}{*}[4,9]{} & \multirow[t]{2}{*}{1} & 0 & 1 & \multirow[t]{2}{*}{$4-27$} & {$[4,6,9,0]$} \\
\hline & & 6 & 7 & & {$[1,4,7,9]$} \\
\hline \multirow[t]{2}{*}[4,9]{} & \multirow[t]{2}{*}{1} & 0 & 1 & \multirow[t]{2}{*}{$4-26$} & {$[4,7,9,0]$} \\
\hline & & 7 & 6 & & {$[1,4,6,9]$} \\
\hline \multirow[t]{2}{*}[6,7]{} & \multirow[t]{2}{*}{1} & 0 & 1 & \multirow[t]{2}{*}{ 4-z29 } & {$[0,4,6,7]$} \\
\hline & & 4 & 9 & & {$[6,7,9,1]$} \\
\hline \multirow[t]{2}{*}[6,7]{} & \multirow[t]{2}{*}{1} & 0 & 1 & \multirow[t]{2}{*}{$4-13$} & {$[6,7,9,0]$} \\
\hline & & 9 & 4 & & {$[1,4,6,7]$} \\
\hline
\end{tabular}

Conjunto 6-z49, (013479)

Alturas $[11,0,2,3,6,8]$

Eixo soma 2, pares $[0,2][3,11][6,8]$

\begin{tabular}{|c|c|c|c|c|c|}
\hline $\begin{array}{c}\text { Pares } \\
(\operatorname{Mod} 2)\end{array}$ & Soma $|\mathbf{s}|$ & Altura $|\mathrm{x}|$ & $\begin{array}{l}\text { Altura }|\mathbf{y}| \\
|\mathbf{y}|=|\mathbf{s}|-|\mathbf{x}|\end{array}$ & Conjunto & Tetracordes \\
\hline \multirow[t]{2}{*}[0,2]{} & \multirow[t]{2}{*}{2} & 3 & 11 & \multirow[t]{2}{*}{$4-12$} & {$[0,2,3,6]$} \\
\hline & & 6 & 8 & & {$[8,11,0,2]$} \\
\hline \multirow[t]{2}{*}[0,2]{} & \multirow[t]{2}{*}{2} & 3 & 11 & \multirow[t]{2}{*}{$4-z 29$} & {$[8,0,2,3]$} \\
\hline & & 8 & 6 & & {$[11,0,2,6]$} \\
\hline \multirow[t]{2}{*}[3,11]{} & \multirow[t]{2}{*}{2} & 6 & 8 & \multirow[t]{2}{*}{$4-18$} & {$[11,0,3,6]$} \\
\hline & & 0 & 2 & & {$[8,11,2,3]$} \\
\hline \multirow[t]{2}{*}[3,11]{} & \multirow[t]{2}{*}{2} & 6 & 8 & \multirow[t]{2}{*}{$4-17$} & {$[11,2,3,6]$} \\
\hline & & 2 & 0 & & {$[8,11,0,3]$} \\
\hline \multirow[t]{2}{*}[6,8]{} & \multirow[t]{2}{*}{2} & 0 & 2 & \multirow[t]{2}{*}{$4-27$} & {$[0,3,6,8]$} \\
\hline & & 3 & 11 & & {$[6,8,11,2]$} \\
\hline \multirow[t]{2}{*}[6,8]{} & \multirow[t]{2}{*}{2} & 0 & 2 & \multirow[t]{2}{*}{$4-z 15$} & {$[6,8,11,0]$} \\
\hline & & 11 & 3 & & {$[2,3,6,8]$} \\
\hline
\end{tabular}


Conjunto 6-z13, (013467)

Alturas $[11,0,2,3,5,6]$

Eixo soma 5, pares $[2,3][0,5][6,11]$

\begin{tabular}{|c|c|c|c|c|c|}
\hline $\begin{array}{c}\text { Pares } \\
(\operatorname{Mod} 2)\end{array}$ & Soma $|s|$ & Altura $|\mathrm{x}|$ & $\begin{array}{l}\text { Altura }|\mathrm{y}| \\
|\mathrm{y}|=|\mathrm{s}|-|\mathrm{x}|\end{array}$ & Conjunto & Tetracordes \\
\hline \multirow[t]{2}{*}[2,3]{} & \multirow[t]{2}{*}{5} & 0 & 5 & \multirow[t]{2}{*}{$4-12$} & {$[0,2,3,6]$} \\
\hline & & 6 & 11 & & {$[11,2,3,5]$} \\
\hline \multirow[t]{2}{*}[2,3]{} & \multirow[t]{2}{*}{5} & 0 & 5 & \multirow[t]{2}{*}{$4-3$} & {$[11,0,2,3]$} \\
\hline & & 11 & 6 & & {$[2,3,5,6]$} \\
\hline \multirow[t]{2}{*}[0,5]{} & \multirow[t]{2}{*}{5} & 2 & 3 & \multirow[t]{2}{*}{$4-z 15$} & {$[0,2,5,6]$} \\
\hline & & 6 & 11 & & {$[11,0,3,5]$} \\
\hline \multirow[t]{2}{*}[0,5]{} & \multirow[t]{2}{*}{5} & 2 & 3 & \multirow[t]{2}{*}{$4-13$} & {$[11,0,2,5]$} \\
\hline & & 11 & 6 & & {$[0,3,5,6]$} \\
\hline \multirow[t]{2}{*}[6,11]{} & \multirow[t]{2}{*}{5} & 0 & 5 & \multirow[t]{2}{*}{$4-z 29$} & {$[11,0,2,6]$} \\
\hline & & 2 & 3 & & {$[11,3,5,6]$} \\
\hline \multirow[t]{2}{*}[6,11]{} & \multirow[t]{2}{*}{5} & 0 & 5 & \multirow[t]{2}{*}{$4-18$} & {$[11,0,3,6]$} \\
\hline & & 3 & 2 & & {$[11,2,5,6]$} \\
\hline
\end{tabular}

Conjunto 6-z6, (012567)

Alturas $[11,0,1,4,5,6]$

Eixo soma 5, pares $[1,4][0,5][6,11]$

\begin{tabular}{|c|c|c|c|c|c|}
\hline $\begin{array}{c}\text { Pares } \\
(\operatorname{Mod} 2)\end{array}$ & Soma $|\mathbf{s}|$ & Altura $|\mathrm{x}|$ & $\begin{array}{l}\text { Altura }|\mathrm{y}| \\
|\mathrm{y}|=|\mathbf{S}|-|\mathbf{x}|\end{array}$ & Conjunto & Tetracordes \\
\hline \multirow[t]{2}{*}[1,4]{} & \multirow[t]{2}{*}{5} & 0 & 5 & \multirow[t]{2}{*}{$4-z 15$} & {$[0,1,4,6]$} \\
\hline & & 6 & 11 & & {$[11,1,4,5]$} \\
\hline \multirow[t]{2}{*}[1,4]{} & \multirow[t]{2}{*}{5} & 0 & 5 & \multirow[t]{2}{*}{$4-4$} & {$[11,0,1,4]$} \\
\hline & & 11 & 6 & & {$[1,4,5,6]$} \\
\hline \multirow[t]{2}{*}[0,5]{} & \multirow[t]{2}{*}{5} & 1 & 4 & \multirow[t]{2}{*}{$4-8$} & {$[0,1,5,6]$} \\
\hline & & 6 & 11 & & {$[11,0,4,5]$} \\
\hline \multirow[t]{2}{*}[0,5]{} & \multirow[t]{2}{*}{5} & 1 & 4 & \multirow[t]{2}{*}{$4-5$} & {$[11,0,1,5]$} \\
\hline & & 11 & 6 & & {$[0,4,5,6]$} \\
\hline \multirow[t]{2}{*}[6,11]{} & \multirow[t]{2}{*}{5} & 0 & 5 & \multirow[t]{2}{*}{$4-6$} & {$[11,0,1,6]$} \\
\hline & & 1 & 4 & & {$[4,5,6,11]$} \\
\hline \multirow[t]{2}{*}[6,11]{} & \multirow[t]{2}{*}{5} & 0 & 5 & \multirow[t]{2}{*}{$4-18$} & {$[11,0,3,6]$} \\
\hline & & 3 & 2 & & {$[11,2,5,6]$} \\
\hline
\end{tabular}


Conjunto 6-z4, (012456)

Alturas $[0,1,2,4,5,6]$

Eixo soma 6, pares $[2,4][1,5][0,6]$

\begin{tabular}{|c|c|c|c|c|c|}
\hline $\begin{array}{c}\text { Pares } \\
(\operatorname{Mod} 2)\end{array}$ & Soma $|s|$ & Altura $|\mathrm{x}|$ & $\begin{array}{l}\text { Altura }|\mathrm{y}| \\
|\mathrm{y}|=|\mathbf{s}|-|\mathrm{x}|\end{array}$ & Conjunto & Tetracordes \\
\hline \multirow[t]{2}{*}[2,4]{} & \multirow[t]{2}{*}{6} & 1 & 5 & \multirow[t]{2}{*}{$4-2$} & {$[0,1,2,4]$} \\
\hline & & 0 & 6 & & {$[2,4,5,6]$} \\
\hline \multirow[t]{2}{*}[2,4]{} & \multirow[t]{2}{*}{6} & 1 & 5 & \multirow[t]{2}{*}{$4-11$} & {$[1,2,4,6]$} \\
\hline & & 6 & 0 & & {$[0,2,4,5]$} \\
\hline \multirow[t]{2}{*}[1,5]{} & \multirow[t]{2}{*}{6} & 2 & 4 & \multirow[t]{2}{*}{$4-4$} & {$[0,1,2,5]$} \\
\hline & & 0 & 6 & & {$[1,4,5,6]$} \\
\hline \multirow[t]{2}{*}[1,5]{} & \multirow[t]{2}{*}{6} & 2 & 4 & \multirow[t]{2}{*}{$4-7$} & {$[1,2,5,6]$} \\
\hline & & 6 & 0 & & {$[0,1,4,5]$} \\
\hline \multirow[t]{2}{*}[0,6]{} & \multirow[t]{2}{*}{6} & 1 & 5 & \multirow[t]{2}{*}{$4-5$} & {$[0,1,2,6]$} \\
\hline & & 2 & 4 & & {$[0,4,5,6]$} \\
\hline \multirow[t]{2}{*}[0,6]{} & \multirow[t]{2}{*}{6} & 1 & 5 & \multirow[t]{2}{*}{$4-z 15$} & {$[0,1,4,6]$} \\
\hline & & 4 & 2 & & {$[0,2,5,6]$} \\
\hline
\end{tabular}

Conjunto 6-z38, (012378)

Alturas $[0,1,2,3,7,8]$

Eixo soma 3, pares $[1,2][0,3][7,8]$

\begin{tabular}{|c|c|c|c|c|c|}
\hline $\begin{array}{c}\text { Pares } \\
(\operatorname{Mod} 2)\end{array}$ & Soma $|\mathbf{s}|$ & Altura $|\mathrm{x}|$ & $\begin{array}{l}\text { Altura }|\mathrm{y}| \\
|\mathrm{y}|=|\mathbf{s}|-|\mathrm{x}|\end{array}$ & Conjunto & Tetracordes \\
\hline \multirow[t]{2}{*}[1,2]{} & \multirow[t]{2}{*}{3} & 0 & 3 & \multirow[t]{2}{*}{$4-6$} & {$[0,1,2,7]$} \\
\hline & & 7 & 8 & & {$[1,2,3,8]$} \\
\hline \multirow[t]{2}{*}[1,2]{} & \multirow[t]{2}{*}{3} & 0 & 3 & \multirow[t]{2}{*}{$4-5$} & {$[8,0,1,2]$} \\
\hline & & 8 & 7 & & {$[1,2,3,7]$} \\
\hline \multirow[t]{2}{*}[0,3]{} & \multirow[t]{2}{*}{3} & 1 & 2 & \multirow[t]{2}{*}{$4-z 29$} & {$[0,1,3,7]$} \\
\hline & & 7 & 8 & & {$[8,0,2,3]$} \\
\hline \multirow[t]{2}{*}[0,3]{} & \multirow[t]{2}{*}{3} & 1 & 2 & \multirow[t]{2}{*}{$4-14$} & {$[8,0,1,3]$} \\
\hline & & 8 & 7 & & {$[0,2,3,7]$} \\
\hline \multirow[t]{2}{*}[7,8]{} & \multirow[t]{2}{*}{3} & 0 & 3 & \multirow[t]{2}{*}{$4-8$} & {$[7,8,0,1]$} \\
\hline & & 1 & 2 & & {$[2,3,7,8]$} \\
\hline \multirow[t]{2}{*}[7,8]{} & \multirow[t]{2}{*}{3} & 0 & 3 & \multirow[t]{2}{*}{$4-16$} & {$[7,8,0,2]$} \\
\hline & & 2 & 1 & & {$[1,3,7,8]$} \\
\hline
\end{tabular}


Conjunto 6-z26, (013578)

Alturas $[0,1,3,5,7,8]$

Eixo soma 8, pares $[3,5][1,7][0,8]$

\begin{tabular}{|c|c|c|c|c|c|}
\hline $\begin{array}{c}\text { Pares } \\
(\operatorname{Mod} 2)\end{array}$ & Soma $|\mathbf{s}|$ & Altura $|\mathrm{x}|$ & $\begin{array}{l}\text { Altura }|\mathrm{y}| \\
|\mathrm{y}|=|\mathbf{s}|-|\mathbf{x}|\end{array}$ & Conjunto & Tetracordes \\
\hline \multirow[t]{2}{*}[3,5]{} & \multirow[t]{2}{*}{8} & 1 & 7 & \multirow[t]{2}{*}{$4-11$} & {$[0,1,3,5]$} \\
\hline & & 0 & 8 & & {$[3,5,7,8]$} \\
\hline \multirow[t]{2}{*}[3,5]{} & \multirow[t]{2}{*}{8} & 1 & 7 & \multirow[t]{2}{*}{$4-22$} & {$[1,3,5,8]$} \\
\hline & & 8 & 0 & & {$[0,3,5,7]$} \\
\hline \multirow[t]{2}{*}[1,7]{} & \multirow[t]{2}{*}{8} & 3 & 5 & \multirow[t]{2}{*}{$4-z 29$} & {$[0,1,3,7]$} \\
\hline & & 0 & 8 & & {$[1,5,7,8]$} \\
\hline \multirow[t]{2}{*}[1,7]{} & \multirow[t]{2}{*}{8} & 3 & 5 & \multirow[t]{2}{*}{$4-16$} & {$[1,3,7,8]$} \\
\hline & & 8 & 0 & & {$[0,1,5,7]$} \\
\hline \multirow[t]{2}{*}[0,8]{} & \multirow[t]{2}{*}{8} & 1 & 7 & \multirow[t]{2}{*}{$4-14$} & {$[8,0,1,3]$} \\
\hline & & 3 & 5 & & {$[5,7,8,0]$} \\
\hline \multirow[t]{2}{*}[0,8]{} & \multirow[t]{2}{*}{8} & 1 & 7 & \multirow[t]{2}{*}{$4-20$} & {$[0,1,5,8]$} \\
\hline & & 5 & 3 & & {$[7,8,0,3]$} \\
\hline
\end{tabular}

Tab. 12: Mapeamento dos seis conjuntos de tetracordes que podem projetar cada um dos dez hexacordes que relacionam os tetracordes 4-27, 4-12, 4-13, 4-18, 4-z15 e 4-z29

A partir deste mapeamento, podemos construir uma rede de projeções por inversão em torno dos seis hexacordes relacionados aos tetracordes 4-z15 e 4-z29 (Fig.9).

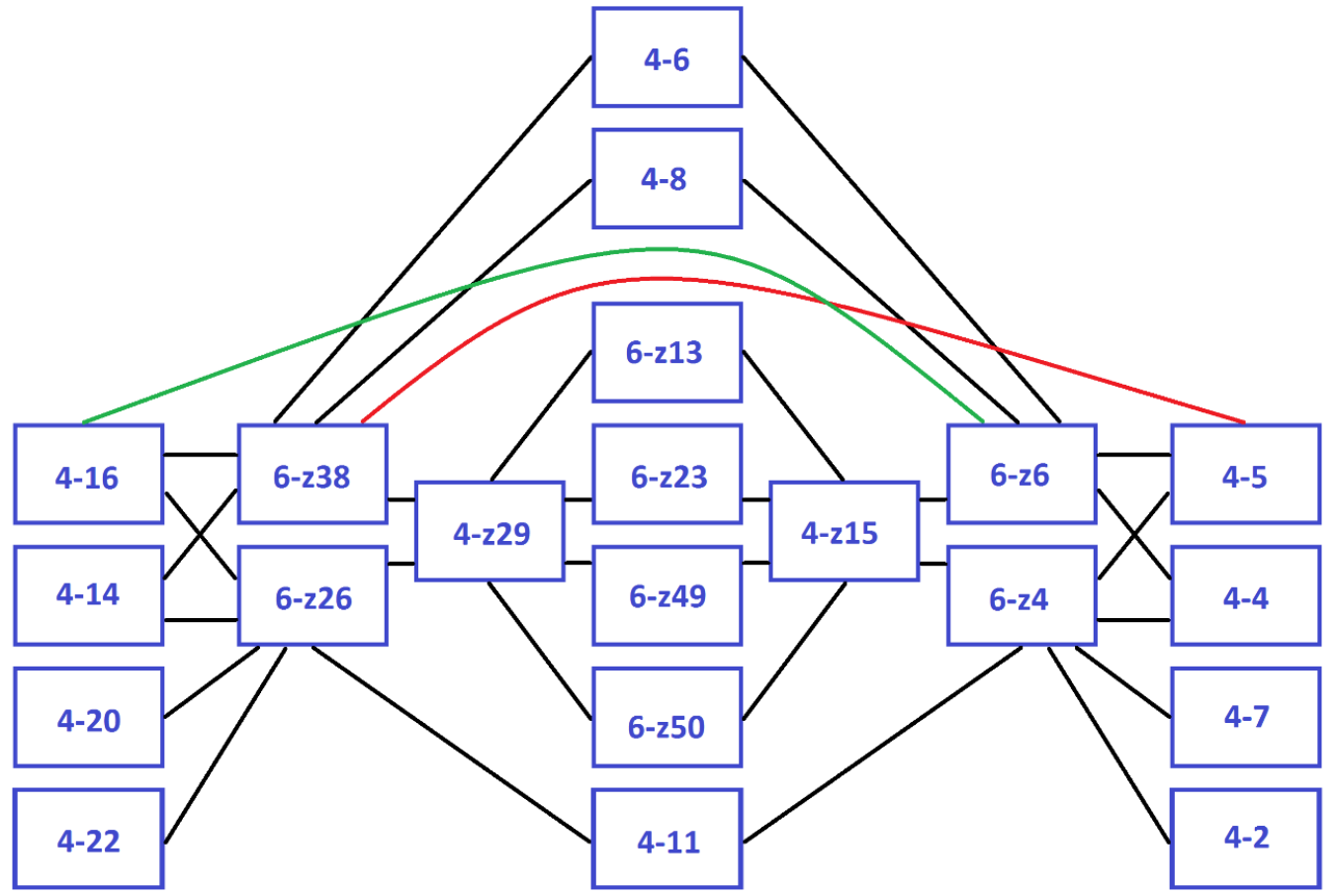

Fig. 9: Rede de projeções por inversão em torno dos seis hexacordes relacionados aos tetracordes 4-z15 e 4-z29 
Novamente temos uma disposição simétrica entre os conjuntos, distribuídos mais uma vez reiterando as regiões $\mathrm{C} 1, \mathrm{C} 5$, central e superior, com correspondência por multiplicação entre os conjuntos das regiões $\mathrm{C} 1$ e C5, como podemos ver na análise dos vetores intervalares dos grupos de alturas destas duas seções ${ }^{3}$. Estas relações foram apresentadas nas figuras a seguir (Fig.10):
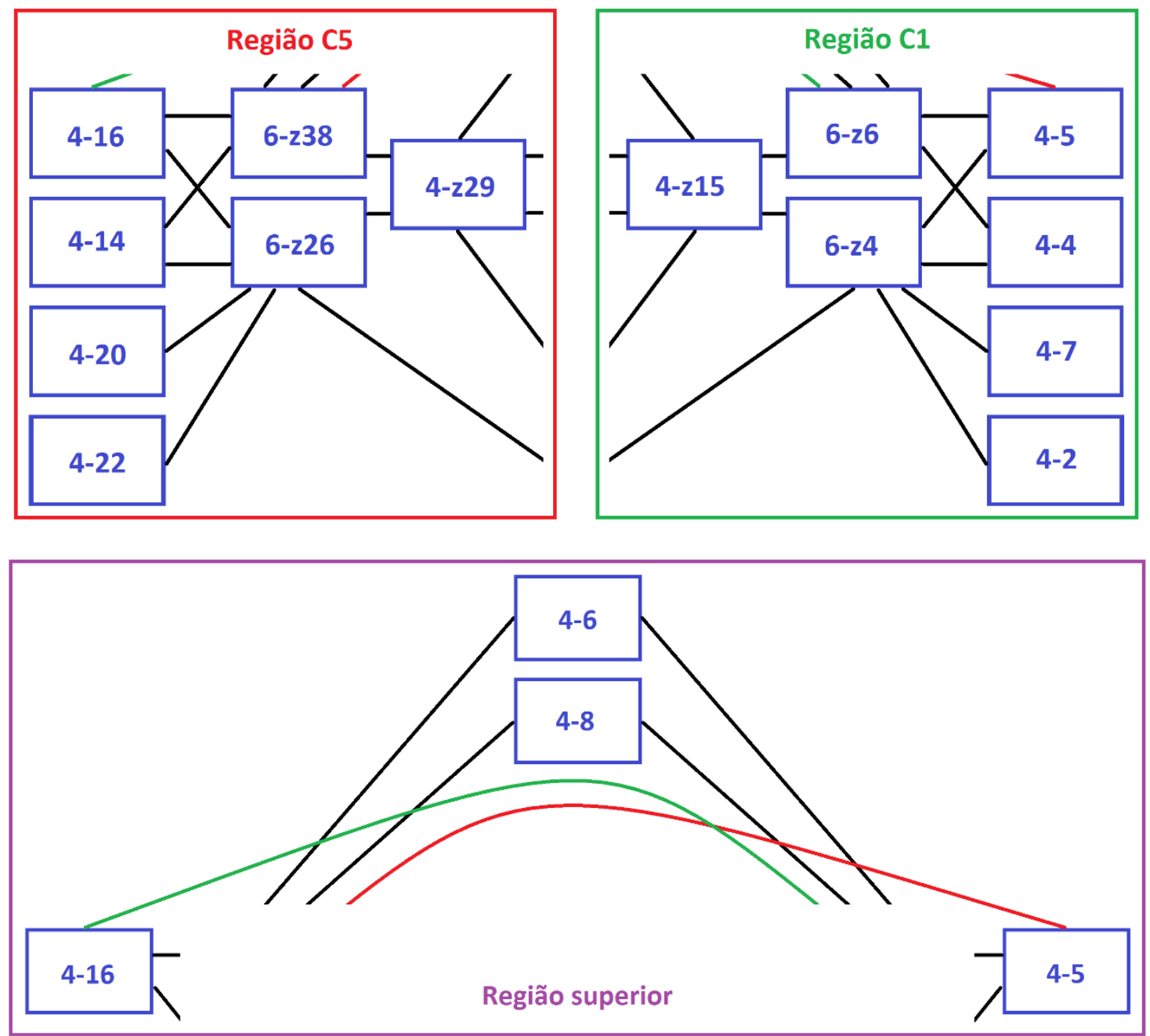
presentes nas regiões C5 e C1 por se relacionarem por multiplicação. Trataremos os conjuntos Z-relacionados como estruturas com multiplicidades iguais nas entradas um e cinco, resultando na reiteração do mesmo índice nestes vetores quando o conjunto é submetido ao processo de multiplicação M5 ou M7. Lembrando que nem todos os conjuntos Z-relacionados estão relacionados por multiplicação M5 ou M7, como aponta Oliveira (2007: 117). 


\section{Região central}

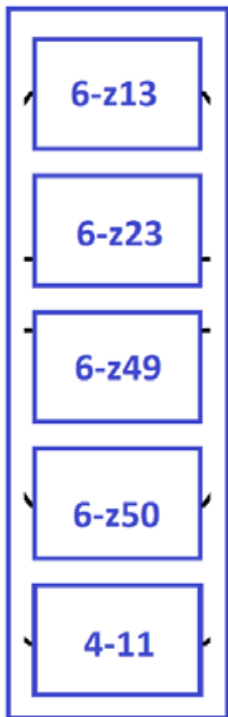

Fig. 10: Disposição simétrica entre os conjuntos, distribuídos reiterando as regiões C1, C5, central e superior

Temos a seguir as relações de multiplicação M5 e M7 entre os conjuntos das regiões C1 e C5, verificada pela análise dos vetores intervalares (Tab.13):

\begin{tabular}{|c|c|c|c|}
\hline Região C5 & Vetores & Região C1 & Vetores \\
\hline $4-z 29$ & {$[111111]$} & $4-z 15$ & {$[111111]$} \\
\hline $4-16$ & {$[110121]$} & $4-5$ & {$[210111]$} \\
\hline $4-14$ & {$[111120]$} & $4-4$ & {$[211110]$} \\
\hline $4-20$ & {$[101220]$} & $4-7$ & {$[201210]$} \\
\hline $4-22$ & {$[021120]$} & $4-2$ & {$[221100]$} \\
\hline $6-z 38$ & {$[421242]$} & $6-z 6$ & {$[421242]$} \\
\hline $6-z 26$ & {$[232341]$} & $6-z 4$ & {$[432321]$} \\
\hline
\end{tabular}

Tab. 13: Relações de multiplicação M5 e M7 entre os conjuntos das regiões C1 e C5

Poderíamos ainda incluir nesta disposição entre região C1 e C5 os hexacordes da região central 6-z50 e 6-z13, pois também apresentam relação de multiplicação pelos fatores M5 e M7, como podemos verificar na análise dos vetores intervalares destes conjuntos (Tab.14): 


\begin{tabular}{|c|c|c|c|}
\hline Região C5 & Vetores & Região C1 & Vetores \\
\hline $6-z 50$ & {$[224232]$} & $6-z 13$ & {$[324222]$} \\
\hline
\end{tabular}

Tab. 14: Relação de multiplicação pelos fatores M5 e M7 entre os hexacordes da região central 6-z50 e 6-z13

Também podemos incluir os tetracordes 4-z15 e 4-z29 à rede de tetracordes relacionados por invariâncias de quatro entradas de vetores intervalares, como podemos verificar na figura abaixo (Fig.11). Interessante perceber que os dois conjuntos incluídos têm o mesmo índice 1 em todas as entradas de vetores, além de cada um poder se relacionar com outros oito tetracordes por invariância de vetores, uma capacidade de interação maior do que qualquer outro conjunto do sistema. Novamente identificamos tetracordes simétricos (s) e assimétricos (a)

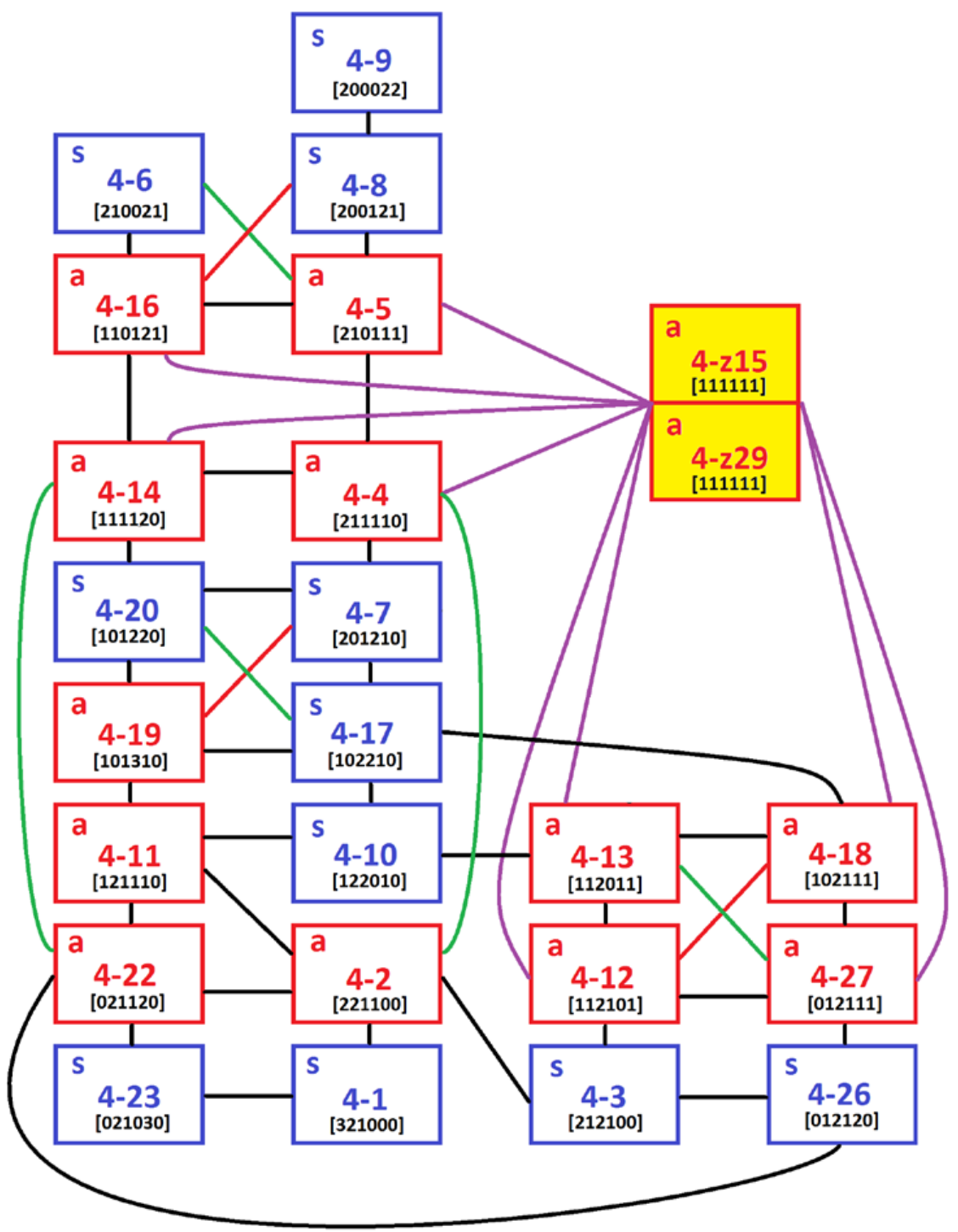

Fig. 11: Rede de tetracordes relacionados por invariâncias de quatro entradas de vetores intervalares, incluindo os conjuntos 
Seguindo o levantamento feito anteriormente, podemos também apresentar uma expansão da rede de projeções por inversão em torno dos seis hexacordes relacionados aos tetracordes 4-27, 4-12, 4-13 e 4-18 ("constelação Morris"). Estas relações foram apresentadas no gráfico a seguir (Fig.12). Temos novamente um sistema simétrico, organizado em duas metades de conjuntos relacionados por multiplicação que compõem as regiões $\mathrm{C} 1$ e $\mathrm{C} 5$, intermediados por um grupo de conjuntos que formam a região central (Fig.13). Referente a região superior temos apenas os conjuntos 4-16 e 4-5, que foram incluídos aqui nas regiões $\mathrm{C} 1$ e C5 por também se relacionarem por multiplicação entre si pelos fatores M5 e M7.

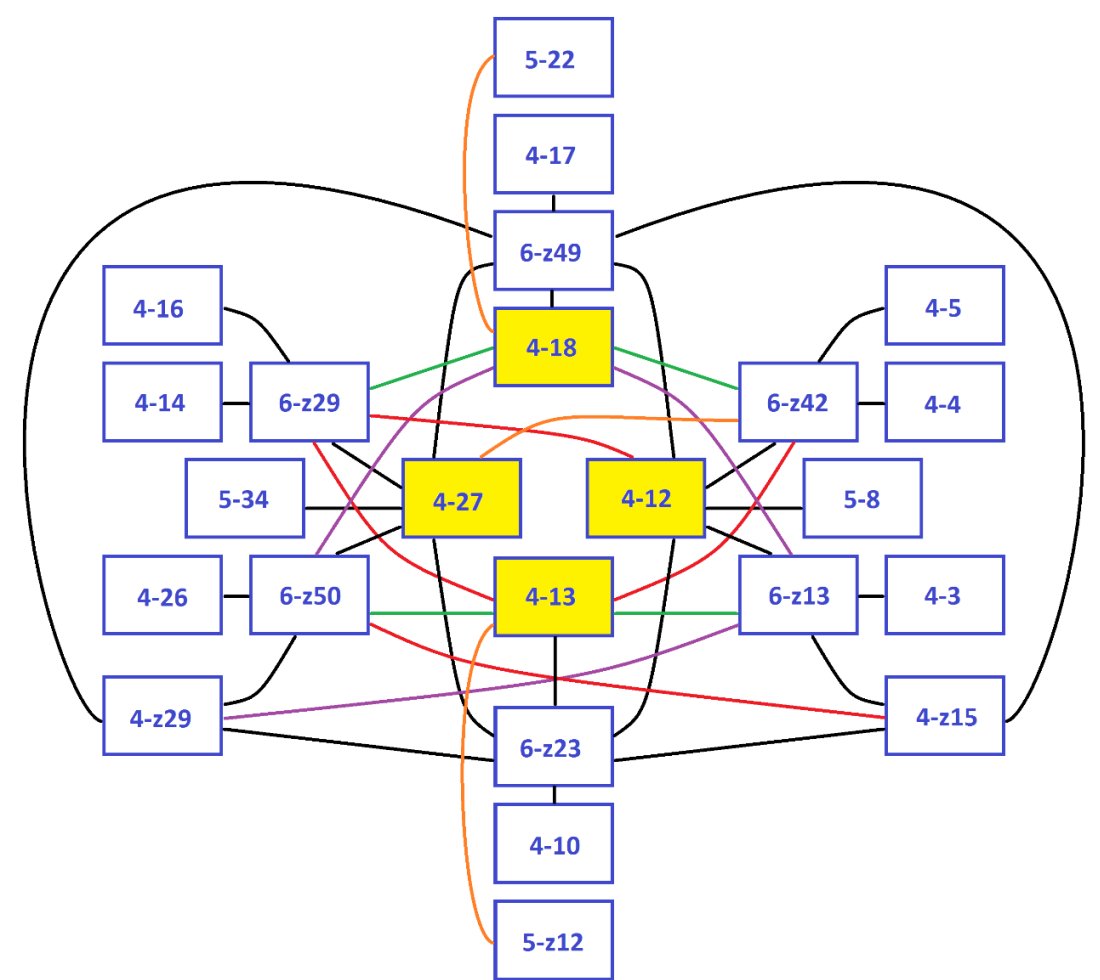

Fig. 12: Rede de projeções por inversão em torno dos seis hexacordes relacionados aos tetracordes 4-27, 4-12, 4-13 e 4-18 ("constelação Morris")
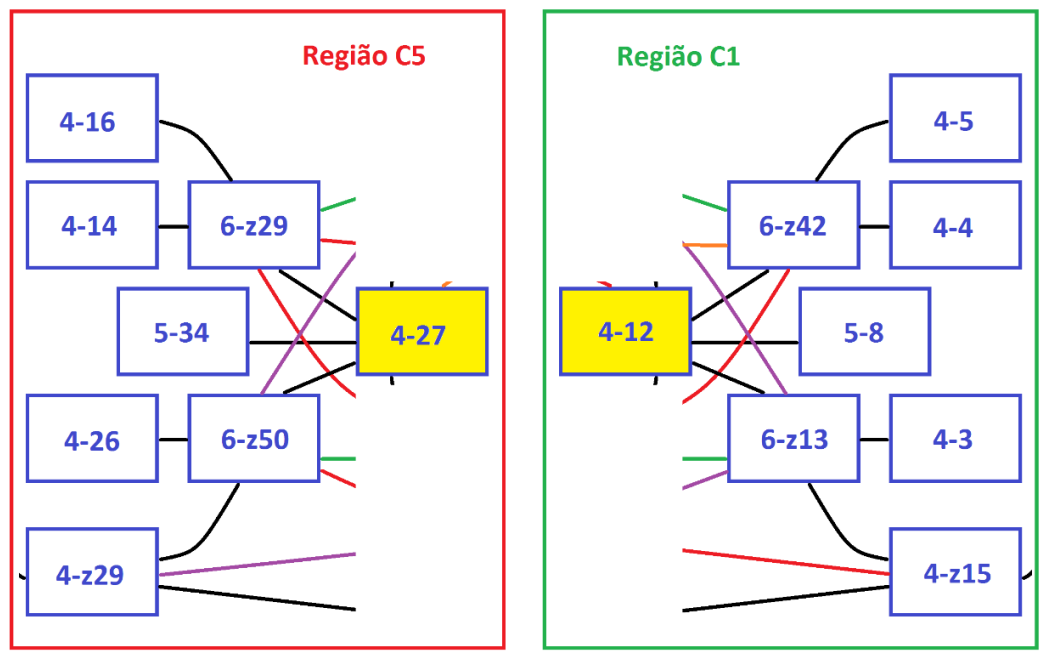


\section{Região central}

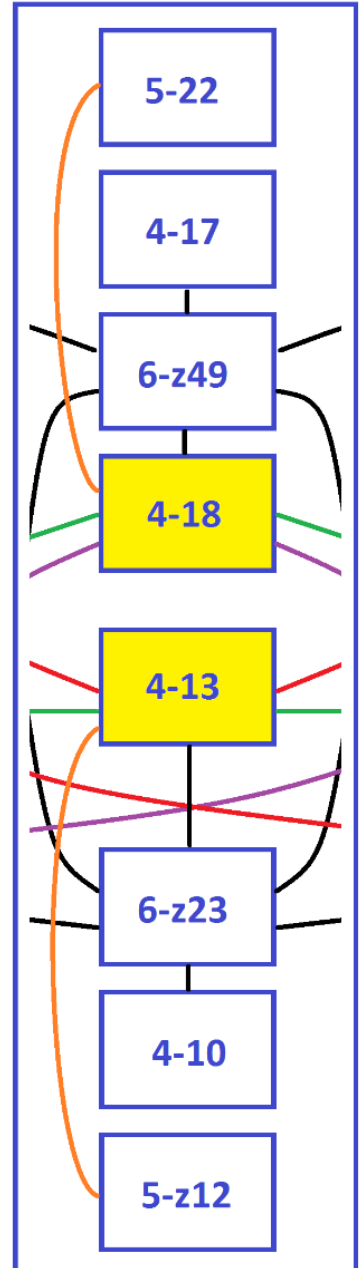

Fig. 13: Disposição simétrica entre os conjuntos, distribuídos reiterando as regiões C1, C5 e Central

Finalmente, podemos reunir todas as conexões entre os tetracordes 4-12, 4-27, 4-18, 4-13, 4-z15 e 4-z29 e os dez hexacordes a estes relacionados, incluindo também todos os outros tetracordes que podem projetar estes hexacordes, além dos quatro pentacordes projetados pelos tetracordes da "constelação Morris". Apresentamos todos estes trinta e cinco conjuntos em um único sistema simétrico de projeções por inversão, como podemos ver a seguir (Fig.14): 


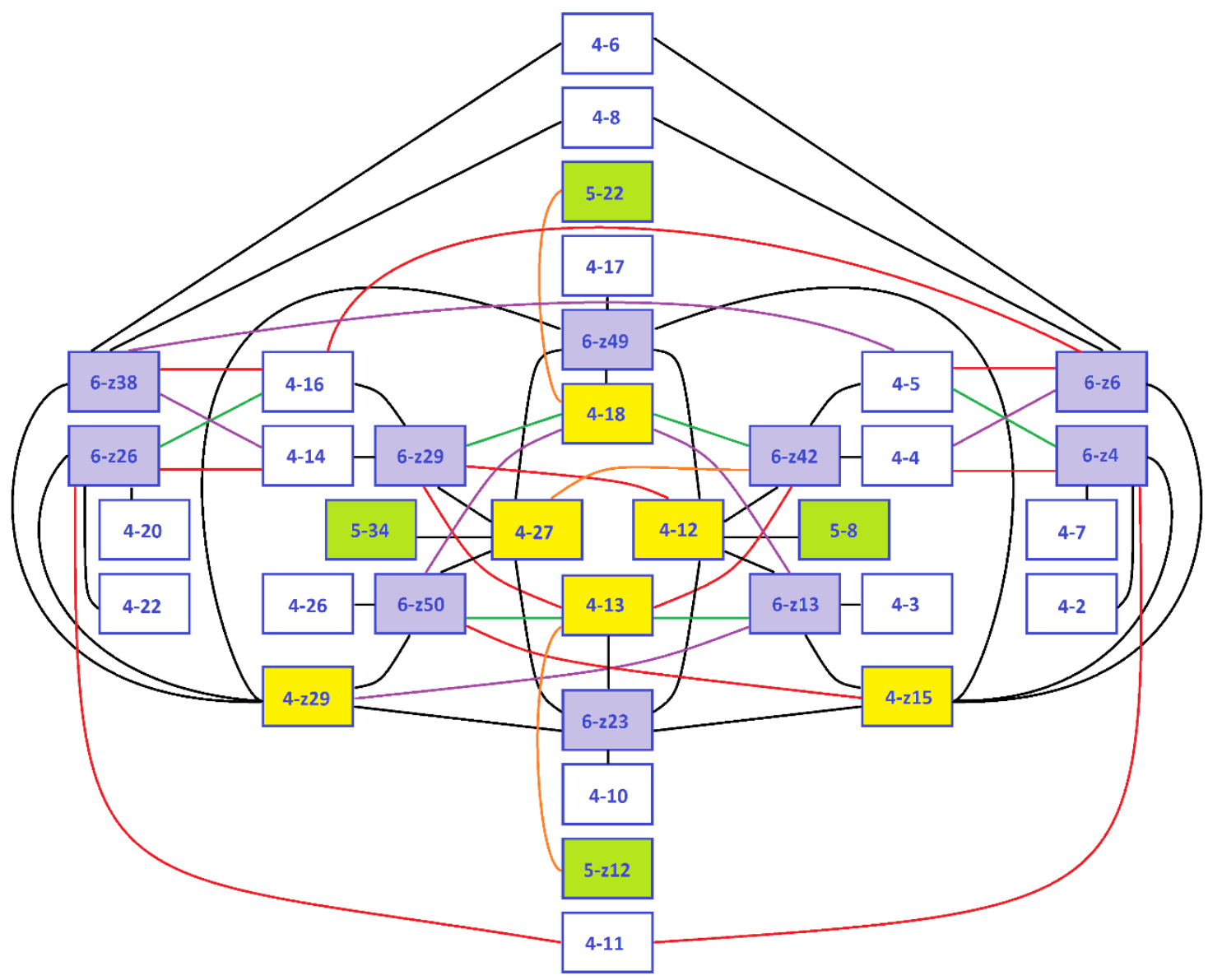

Fig. 14: Rede de projeções por inversão a partir dos tetracordes 4-12, 4-27, 4-18, 4-13, 4-z15 e 4-z29

Acreditamos que a rede de projeções por inversão - conceito desenvolvido a partir de aprofundamentos sobre a teoria neorriemanniana, partindo da perspectiva de Lewin (1982) - encontra correspondências em estudos desenvolvidos por musicólogos inscritos em outra corrente teórica que se dedica ao universo pós-tonal: a teoria dos ciclos intervalares de George Perle (1977). Seguindo esta constatação, corroboramos a suposição levantada por Robert Morris (2007) de que as principais correntes de estudo sobre a teoria pós-tonal - teoria neorriemanniana (LEWIN), teoria dos conjuntos (FORTE) e teoria dos ciclos (PERLE) - poderiam ser alinhadas em torno de uma disposição comum inerente ao sistema de doze notas, possível ser mapeado utilizando a teoria dos grupos - segmento da matemática, especializado no estudo de dimensões simétricas através de estruturas algébricas (matrizes).

\section{Conclusão}

Este presente trabalho tomou como objetivo a averiguação de dimensões simétricas entre classes de intervalos inerentes ao próprio sistema de doze alturas, fatores de organização harmônica que estão sendo aplicadas em nossas análises de obras pós-tonais do início do século XX e afins. Sublinhamos aqui a importância do trabalho desenvolvido por pesquisadores empenhados em desenvolver estratégias teóricas voltadas 
para a análise de obras que recorrem a ferramentas harmônicas pós-tonais, dos quais foram listados em nossa bibliografia alguns dos textos mais relevantes para realização deste artigo. Entre estas diversas propostas que foram sendo aprimoradas ao longo das últimas décadas, selecionamos aqui alguns instrumentos oriundos de uma destas vertentes metodológicas - a teoria neorriemanniana, na qual este presente trabalho se inscreve - com destaque para a rede de alturas (Tonnetz) a partir de qualquer classe de conjunto, seguindo uma suposição apresentada por Lewin (1982).

No campo de estudos da teoria neorriemanniana, desenvolvemos uma extensa reflexão a partir deste conceito de rede de alturas (Tonnetz), apresentando as diversas possibilidades de construção de Tonnetze a partir todos os tricordes e tetracordes previstos. Além disso, percebemos relações de invariância entre estes diferentes Tonnetze, parâmetros que foram alinhados em um sistema maior e mais amplo que chamamos neste trabalho de rede de projeções por inversões, abrindo espaço para um novo segmento de estudo dentre do campo da teoria neorriemanniana - que chamamos aqui de teoria da inversão. O ponto de partida desta série de especulações nasceu de uma hipótese levantada por Robert Morris (2007), o qual constatou que poderia haver um fator de alinhamento entre as principais correntes de estudo dedicadas ao universo pós-tonal - teoria dos conjuntos (Forte), teoria neorriemanniana (Lewin) e teoria dos ciclos intervalares (Perle) - em torno de um mesmo princípio de organização harmônica. Mais do que isso, Morris enfatiza que este alinhando estre estas correntes teóricas poderia ser demonstrada através da teoria dos grupos - um segmento de estudo oriundo da matemática, a qual se dedica ao estudo da simetria utilizando estruturas algébricas conhecidas como matrizes. Esta suposição do autor foi tomada neste trabalho e corroborada ao longo de nossa pesquisa, culminando no desenvolvimento das diferentes perspectivas de construção de segmentos da rede de projeções por inversão apresentadas ao longo do texto. Reconhecemos que não esgotamos ainda todos os caminhos de projeções possíveis que devem existir neste sistema que construímos, mas acreditamos que a rede de projeções por inversão alcance todos as classes de alturas previstos no sistema de doze notas, revelando assim esta suposição prevista no texto de Morris (2007).

Notamos na rede de projeções por inversão uma propriedade simétrica inerente ao sistema cromático, constatando uma dimensão proporcional que envolve todas as classes de alturas do universo das doze notas. Além disso, construímos um sistema que relaciona conjuntos de diferentes cardinalidades, possibilidade não prevista em outra vertente descendente da teoria neorriemanniana - a teoria transformacional, que gravita principalmente em torno do trabalho de Richard Cohn e Tymoczko - que se empenha na construção de redes de conjuntos com um mesmo número de alturas, com destaque para a "dança dos cubos" com tricordes e o sistema com "torres" de tetracordes (COHN, 2012). Em nossa proposta de análise de obras pós-tonais, mostrou-se necessário a construção de sistemas que relacionassem conjuntos de qualquer ordem e de diferentes espécies, não apenas os convencionais conjuntos 3-11 e 4-27 trabalhados a exaustão nas teorias neorriemanniana tradicional e teoria transformacional. Desta forma, se revelou extremamente eficiente a nossa proposta de construção desta rede de projeções por inversão e sua utilização no mapeamento de estruturas intervalares de obras pós-tonais. 
Percebemos ainda que a rede de projeções por inversão pode ser segmentada em setores harmônicos correlacionados (com destaque paras as regiões C1 e C5, correspondentes por multiplicação pelos fatores M5 e M7). Desta forma, dependendo da espécie de conjuntos recorrentes em determinado trecho de uma obra analisada, podemos determinar o perfil harmônico preferido por um compositor em um determinado contexto. Esta verificação pode ainda ser estendida e corroborada ao incluirmos um número maior de exemplos de um mesmo compositor, revelando seus gostos e predileções dentro da rede de projeções por inversão.

\section{Bibliografia}

ALBUQUERQUE, Joel; SALLES, Paulo de Tarso. "Rede de Projeções por Inversão, Relações entre Tonnetze de Diferentes Tricordes". MUSICA THEORICA. Salvador: TeMA, 2017, p. 139-163.

ANTOKOLETZ, Elliott. La Música de Béla Bartók: un estudio de la tonalidad y la progresión en la música Del siglo XX. Traduzido para o espanhol por José Ángel García Corona. Barcelona: Idea Books, S.A., 2006.

BABBITT, Milton. "Twelve-Tone Invariants as Compositional Determinants". The Musical Quarterly, Vol. 46, No. 2, Special Issue: Problems of Modern Music. The Princeton Seminar in Advanced Musical Studies (Apr., 1960), pp. 246-259

"Set Structure as a Compositional Determinant". Journal of Music Theory, Vol. 5, No. 1 (Spring, 1961), pp. 72-94.

BOULEZ, Pierre. A música hoje [1963]. 3a ed. Trad. Reginaldo de Carvalho e Mary A. L. de Barros. Editora Perspectiva: 2011.

COHN, Richard. "Inversional Symmetry and Transpositional Combination in Bartók" Music Theory Spectrum, Vol. 10, 10th Anniversary Issue (Spring, 1988), pp. 19-42.

"Introduction to Neo-Riemannian Theory: A Survey and a Historical Perspective". Journal of Music Theory, Vol. 42, No. 2. Neo-Riemannian Theory, p. 167180, Autumn, 1998a.

"Square Dances with Cubes". Journal of Music Theory, Vol. 42, No. 2. Neo-Riemannian Theory, p. 283-296, Autumn, 1998 b.

Audacious Euphony: Chromatic Harmony and the Triad's Second Nature. Nova lorque: Oxford University Press, 2012. 
DOUTHETT, Jack; STEINBACH, Peter. "Parsimonious Graphs: A Study in Parsimony, Contextual Transformations and Modes of Limited Transposition". Journal of Music Theory, Vol. 42, No. 2, Neo-Riemannian Theory, p.241-263, Autumn, 1998.

FOLEY, Gretchen. "Pitch and interval structures in George Perle's Theory of Twelve-tone Tonality". Dissertação de Doutorado em Filosofia pela The University of Western Ontario. Londres, Ontario: 1998.

FORTE, Allen. "Pitch-Class Set Genera and the Origin of Modern Harmonic Species". Journal of Music Theory, Vol. 32, No. 2 (Autumn), p. 187-270, 1988.

GOLLIN, Edward. "Some Aspects of Three-Dimensional Tonnetze". Journal of Music Theory. Vol. 42, n2, Neo-Riemannian Theory (Autumn, 1998), 195-206.

HICKEN, Kenneth. "Tonal Organization in Schoenberg's. Six Little Piano Pieces, Op. 19". Canadian University Music Review / Revue de musique des universités canadiennes, №1, 1980, p. 130-146.

HYDE, Martha. "Twentieth-Century Analysis during the Past Decade". Music Theory Spectrum, Vol. 11, No. 1. The Society for Music Theory: The First Decade, p. 35-39, Spring, 1989.

Journal of Music Theory Vol. 42, No. 2, Neo-Riemannian Theory p.167-348, 1998.

LEWIN, David. "A Formal Theory of Generalized Tonal Functions". Journal of Music Theory, Vol. 26, No. 1 (Spring, 1982), pp. 23-60

Generalized Musical Intervals and Transformations. New Haven: Yale University Press, 1987.

MEAD, Andrew. "The State of Research in Twelve-Tone and Atonal Theory". Music Theory Spectrum, Vol. 11, No. 1, The Society for Music Theory: The First Decade, p. 40-48, Spring, 1989.

MORRIS, Robert. "Review" about Basic Atonal Theory (1980) by John Rahn. Music Theory Spectrum, Vol. 4 (Spring, 1982), pp. 138-154

"Mathematics and the Twelve-Tone System: Past, Present, and Future". Perspectives of New Music, Vol. 45, No. 2 (Summer, 2007), pp. 76-107.

OLIVEIRA, João P. Teoria analítica da música do século XX. 2ª Ed. Lisboa: Calouste Gulbenkian, 2007.

PARKS, Richard. The Music of Claude Debussy. New Haven: Yale University Press, 1989. "Pitch-Class Set Genera: My Theory, Forte's Theory." Music Analysis 17,

p. 206-26, 1998. 
PERLE, George. Twelve-tone Tonality. University of California Press, California: 1977. Twelve-tone Tonality. 2nd. ed. University of California Press, California: 1996.

RAHN, Jay. "Coordination Of Interval Sizes In Seven-Tone Collections", Journal of Music Theory, Vol. 35, No. 1/2 (Spring - Autumn), p. 33-60, 1991.

RAHN, John. Basic Atonal Theory. New York, Longman: 1980.

SALLES, Paulo de Tarso. Villa-Lobos: Processos Composicionais. Campinas, SP: Editora da Unicamp, 2009.

SALLMEN, Mark. "Exploring Tetrachordal Voice-Leading Spaces Within and Around the MORRIS Constellation". Society for Music Theory. Volume 17, Number 4, December 2011.

SCOTTO, Ciro. "Reexamining PC-Set Multiplication, Complex Multiplication, and Transpositional Combination to Determine Their Formal and Functional Equivalence". Perspectives of New Music, Vol. 52, No. 1 (Winter 2014), pp. 134-216

SOLOMON, Larry. "The Table of Pitch Class Sets", 2005. Disponível em < http://solomonsmusic.net/pcsets.htm>

STRAUS, Joseph N. Introduction to Post Tonal Theory. 3a ed. Upper Saddle River: Prentice-Hall, 2005.

STEWART, Ian. Uma História da Simetria na Matemática, Ed. Brasileira. Rio de Janeiro/RJ: Jorge Zahar Editora Ltda, 2012.

TYMOCZKO, Dmitri. "Scale Networks in Debussy", Journal of Music Theory 48(2). 2007: p.215-92.

. "Generalizing Musical Intervals". Journal of Music Theory 53:2, Fall

2009.

A Geometry of Music: Harmony and counterpoint in the extended common practice. New York, NY: Oxford University Press, Inc., 2011.

YUST, Jason. "Applications of DFT to the Theory of Twentieth-Century Harmony". T. Collins et al. (Eds.): MCM 2015, LNAI 9110, pp. 207-218. London: Springer International Publishing, 2015. 